\title{
A MULTICOMPONENT MODEL FOR BIOFILM-DRUG INTERACTION
}

\author{
BRANDON LINDLEY \\ US Naval Research Laboratory \\ 4555 Overlook Ave. Southwest \\ Washington, DC 20375, USA \\ QI WANG \\ Department of Mathematics, Interdisciplinary Mathematics Institute \\ and NanoCenter at USC \\ University of South Carolina \\ Columbia, SC 29208, USA \\ TIANYU ZHANG \\ Department of Mathematical Sciences \\ Montana State University \\ P.O. Box 172400 \\ Bozeman, MT 59717-2400, USA
}

\begin{abstract}
We develop a tri-component model for the biofilm and solvent mixture, in which the extracellular polymeric substance (EPS) network, bacteria and effective solvent consisting of the solvent, nutrient, drugs etc. are modeled explicitly. The tri-component mixture is assumed incompressible as a whole while inter-component mixing, dissipation, and conversion are allowed. A linear stability analysis is conducted on constant equilibria revealing up to two unstable modes corresponding to possible bacterial growth induced by the bacterial and EPS production and dependent upon the regime of the model parameters. A 1-D transient simulation is carried out to investigate the nonlinear dynamics of the EPS network, bacteria distribution, drug and nutrient distribution in a channel with and without shear. Finally, the transient biofilm dynamics are studied with respect to a host of diffusive properties of the drug and nutrient present in the biofilm.
\end{abstract}

1. Introduction. Biofilms are common biological organisms existing in the damp environment. Biofilm forms when bacteria adhere to surfaces in the moist environment by excreting a slimy, glue-like substance called the extracellular polymeric substance (EPS). Sites for biofilm formation include all kinds of surfaces: natural materials above and below ground, metals, plastics, medical implant materials,

2000 Mathematics Subject Classification. Primary: 92C05, 92C15; Secondary: 92B05.

Key words and phrases. Biofilms, Multicomponent fluids, Phase-field model, Steady states, Linearized Stability, Nonlinear Dynamics.

Brandon Lindley and Qi Wang's research is partially supported by the National Science Foundation through grants DMS-0605029, DMS-0626180, EPS-0447660, DMS-0819051, DMS-0908330, and the International Cooperation and Exchanges Program of NSFC 10811120281 and NSFC grant 10601045. They also acknowledge the hospitality during their visit to Chern Institute of Mathematics at Nankai University and Nankai Institute for Scientific Computing in the summer of 2009. Tianyu Zhang's research is partially supported by the NSF grant DMS-0934696 and startup fund from MSU. 
even plant and body tissues. Wherever you find a combination of moisture, bacteria, nutrients and a surface, you are likely to find biofilms there.

Modeling biofilms has been a challenging task. A host of mathematical models have been proposed that yield qualitatively acceptable results in the past. These include low dimensional models primarily focused on steady states [22, 23, 21, 25, $26,14]$, discrete-continuum models coupled with automota $[7,1,2,27,11,20,2,19$, $12,16,17,18]$, multi-fluid models $[16,17,18,8,9,6,15]$, and our own binary phase field model $[28,29]$. For a detailed account of the mathematical models for the biofilm, please refer to our recent review article on the very subject [24]. Among all the models developed so far, very few have separated the EPS network from the bacterial population in highly heterogeneous biofilm regions. However, the microscopic imaging of biofilms shows clearly the distribution of bacteria and EPS networks in a highly heterogeneous microscopical structure. It hence suggests strongly that these two species need to be modeled explicitly in any refined biofilm models. In this paper, we propose a general framework for multi-component materials that can handle dynamical interaction among multiple species and their inter-conversion, dissipation and mixing. In this formulation, the entire material system is assumed incompressible. So, when the volume fraction of one species grows, one or more of the others must be effectively converted into the growing species.

We first recall the binary theory for biofilms developed by Zhang et al. [28], based on which the current formulation is developed. Let $\phi_{n}$ be the volume fraction of the effective polymer network solution consisting of the EPS network and the bacteria, $\phi_{s}$ the one for the effective solvent consisting of the rest of the materials in the biofilm, $\mathbf{v}_{n}$ the velocity of the effective polymer, $\mathbf{v}_{s}$ the velocity of the effective solvent, $c$ the concentration of the substrate, $B$ the concentration of the bacteria, $p$ the hydrostatic pressure. In this model, the nutrient substrate is passively treated as a part of the effective solvent and the bacteria are regarded as a solvent part of the effective polymer. This is a one-fluid two-component biofilm model consisting of following conservation equations.

\section{Momentum and continuity equation}

$$
\begin{aligned}
& \nabla \cdot \mathbf{v}=0, \\
& \rho \frac{d \mathbf{v}}{d t}=\nabla \cdot\left(\phi_{n} \tau_{n}+\phi_{s} \tau_{s}\right)-\left[\nabla p+\gamma_{1} k_{B} T \nabla \cdot\left(\nabla \phi_{n} \nabla \phi_{n}\right)\right],
\end{aligned}
$$

where $\mathbf{v}=\phi_{s} \mathbf{v}_{s}+\phi_{n} \mathbf{v}_{n}$ is the volume-averaged velocity, $\rho=\phi_{n} \rho_{n}+\phi_{s} \rho_{s}$ is the effective density for the mixture, $\rho_{n}$ and $\rho_{s}$ are the density for the effective polymer and solvent, respectively, $\tau_{n}$ and $\tau_{s}$ are the extra stress for the effective polymer and solvent, respectively, $k_{B}$ is the Boltzmann constant, $T$ is the temperature, $\gamma_{1}$ is a parameter measures the strength of the conformation entropy. For the intermixing of the small molecule solvent with the larger molecule effective polymers, we adopt the extended Flory-Huggin's mixing free energy density defined by

$$
f=\frac{\gamma_{1}}{2} k T\left\|\nabla \phi_{n}\right\|^{2}+\gamma_{2} k T\left[\frac{\phi_{n}}{N} \ln \phi_{n}+\left(1-\phi_{n}\right) \ln \left(1-\phi_{n}\right)+\chi \phi_{n}\left(1-\phi_{n}\right)\right],
$$

where $\gamma_{2}$ is the strength of the bulk mixing free energy proportional to the reciprocal of the volume of the solvent molecule, $N$ is the nominal polymerization index for the polymer strand in the effective polymer network and $\chi$ is the mixing parameter [10]. We note that

$$
\phi_{s}+\phi_{n}=1
$$


for the incompressible mixture.

\section{Transport equation for nutrient substrates}

There are two transport models for the nutrient depending on what the transport velocity is assumed.

$$
\begin{array}{ll}
\frac{\partial}{\partial t}\left(\phi_{s} c\right)+\nabla \cdot\left(c \mathbf{v} \phi_{s}-D_{s} \phi_{s} \nabla c\right)=-g_{c}, & \text { CA-model, } \\
\frac{\partial}{\partial t}\left(\phi_{s} c\right)+\nabla \cdot\left(c \mathbf{v}_{s} \phi_{s}-D_{s} \phi_{s} \nabla c\right)=-g_{c}, & \text { CN-model, }
\end{array}
$$

where $c$ is the nutrient concentration and the nutrient consumption rate is given by

$$
g_{c}=\frac{\phi_{n} A c}{K_{0}+c},
$$

$A$ is the maximum nutrient consumption rate, $K_{0}$ is the half saturation constant, and $D_{s}$ is the diffusion constant for the nutrient substrate. The suffix $\mathrm{A}$ and $\mathrm{N}$ in $\mathrm{CA}$ - and $\mathrm{CN}$ - indicate either average $(\mathbf{v})$ or solvent $\left(\mathbf{v}_{s}\right.$ defined below) velocity are used in convection in the transport equation for $c$.

\section{Transport equation for the volume fraction of the polymer network}

$$
\frac{\partial \phi_{n}}{\partial t}+\nabla \cdot\left(\phi_{n} \mathbf{v}\right)=\nabla \cdot\left[\lambda\left(\phi_{n}\right) \nabla \frac{\delta f}{\delta \phi_{n}}\right]+g_{n},
$$

where $\lambda\left(\phi_{n}\right)$ is the mobility parameter and the polymer production rate is given by

$$
g_{n}=\mu \phi_{n} \frac{c}{K_{c}+c},
$$

$\mu$ is the maximum production rate, $K_{c}$ is the half-saturation constant. When $\lambda$ is a constant, the equation is a Cahn-Hilliard equation; while $\lambda$ is a function of $\phi_{n}$ in the form $\lambda=\lambda_{s} \phi_{n}$ or $\lambda=\lambda_{s} \phi_{n}\left(1-\phi_{n}\right)$ with $\lambda_{s}$ a constant, it is a modified or singular Cahn-Hilliard equation with a mobility depending on the polymer volume fraction.

\section{Constitutive equations}

We assume the EPS network obeys a power-law for non-Newtonian fluids:

$$
\begin{array}{lll}
\tau_{n}=2 \eta_{n} \mathbf{D}, & \tau_{s}=2 \eta_{s} \mathbf{D}, & \text { VA-model } \\
\tau_{n}=2 \eta_{n} \mathbf{D}_{n}, & \tau_{s}=2 \eta_{s} \mathbf{D}_{s}, & \text { VN-model }
\end{array}
$$

where $\eta_{n}$ and $\eta_{s}$ are the viscosity for polymer network and solvent, respectively. $\eta_{s}$ is a constant while $\eta_{n}$ is function of the velocity gradient.

$$
\eta_{n}=\eta_{\infty}+\left(\eta_{0}-\eta_{\infty}\right)\left[1+(\lambda \dot{\gamma})^{a}\right]^{(n-1) / a},
$$

where $\dot{\gamma}=\sqrt{\frac{1}{2} \mathbf{D}: \mathbf{D}}, \eta_{0}, \eta_{\infty}, \lambda, a, n$ are five parameters.

The effective polymer velocity in the region where the polymer is present $\left(\phi_{n} \neq 0\right)$ is defined by

$$
\mathbf{v}_{n}=\mathbf{v}-\frac{\lambda}{\phi_{n}} \nabla \frac{\delta f}{\delta \phi_{n}} .
$$

The solvent velocity is given by

$$
\mathbf{v}_{s}=\mathbf{v}+\frac{\lambda}{\phi_{s}} \nabla \frac{\delta f}{\delta \phi_{n}} .
$$

We note that the biofilm is consisted of mainly water so that $\phi_{s} \neq 0$. 
If we use the average velocity to transport the nutrient and the effective polymer, the rate of strain tensor and the vorticity tensor with respect to the average velocity are adopted:

$$
\mathbf{D}=\frac{1}{2}\left[\nabla \mathbf{v}+\nabla \mathbf{v}^{T}\right], \quad \mathbf{W}=\frac{1}{2}\left[\nabla \mathbf{v}-\nabla \mathbf{v}^{T}\right] .
$$

The corresponding ones with respect to the effective polymer velocity are defined analogously

$$
\mathbf{D}_{n}=\frac{1}{2}\left[\nabla \mathbf{v}_{n}+\nabla \mathbf{v}_{n}^{T}\right], \quad \mathbf{W}_{n}=\frac{1}{2}\left[\nabla \mathbf{v}_{n}-\nabla \mathbf{v}_{n}^{T}\right] .
$$

These will be used in the situation where the transport is assumed to be carried out by the polymer velocity. A dumbbell model for the strand of the EPS network and a network theory has also been developed to account for the viscoelastic properties of the effective polymer solution [28]. We omit the details here. Interested readers please refer to [28]. We remark that the bacteria in this simple hydrodynamic model for biofilms is passively treated as a part of the effective polymer solution. Its transport equation, i.e., the transport equation for $B$, is slaved to the equation for the effective EPS and therefore neglected in the model.

This binary model gives two basic growth mechanisms for the biofilm: nutrient fueled growth of the effective polymer and intermixing of the polymer and the solvent. It has been used in simulating biofilm-flow interaction recently [28, 29]. Considering the predominant role of bacteria in biofilms, we next extend this model to account for the dynamics of bacteria explicitly.

2. A ternary theory for biofilms. We consider extensions of the binary model to a single fluid three-component model, named the ternary model, for biofilms. In this theory, we model the EPS and the bacterium as two separate components, respectively, while the nutrient, drugs or disinfectants, and solvent are still collectively modeled as an effective solvent. The diffusive effect of the nutrient and the drug is singled out and modeled explicitly in the solvent. In this model, we denote the volume fraction of the EPS polymer network strand by $\phi_{n}$, that of the bacteria by $\phi_{b}$ and that of the solvent by $\phi_{s}$, respectively.

2.1. Transport equation for each component. We treat the entire material system as an incompressible single fluid mixture consisting of three components: the EPS polymer network, the bacteria, and the effective solvent. We adopt a volume averaged velocity $\mathbf{v}=\phi_{n} \mathbf{v}_{n}+\phi_{b} \mathbf{v}_{b}+\phi_{s} \mathbf{v}_{s}$ to describe the macroscopic transport of the mixture. We then modify the transport of the volume fraction for $\phi_{n}, \phi_{b}$ and $\phi_{s}$ by accounting the average velocity and an additional velocity for each component, due to the mixing free energy, called the excessive velocity for that component:

$$
\begin{aligned}
& \frac{\partial \phi_{n}}{\partial t}+\nabla \cdot\left(\phi_{n} \mathbf{v}\right)=\nabla \cdot\left[\alpha_{11} \nabla \frac{\delta f}{\delta \phi_{n}}+\alpha_{12} \nabla \frac{\delta f}{\delta \phi_{b}}+\alpha_{13} \nabla \frac{\delta f}{\delta \phi_{s}}\right]+g_{n b}, \\
& \frac{\partial \phi_{b}}{\partial t}+\nabla \cdot\left(\phi_{b} \mathbf{v}\right)=\nabla \cdot\left[\alpha_{21} \nabla \frac{\delta f}{\delta \phi_{n}}+\alpha_{22} \nabla \frac{\delta f}{\delta \phi_{b}}+\alpha_{23} \nabla \frac{\delta f}{\delta \phi_{s}}\right]+g_{s b}, \\
& \frac{\partial \phi_{s}}{\partial t}+\nabla \cdot\left(\phi_{s} \mathbf{v}\right)=\nabla \cdot\left[\alpha_{31} \nabla \frac{\delta f}{\delta \phi_{n}}+\alpha_{32} \nabla \frac{\delta f}{\delta \phi_{b}}+\alpha_{33} \nabla \frac{\delta f}{\delta \phi_{s}}\right]+g_{s},
\end{aligned}
$$

where $g_{n b}$ is the reaction rate for the creation of the strand in the EPS polymer network due to the presence of bacteria, $g_{s b}$ is the reaction rate for the bacteria due to the substrate materials (including nutrient and drug) as well as bacteria reproduction, and $g_{s}$ is the reaction rate for the decrease of the substrate materials 
in the effective solvent. The coefficient matrix $\left(\alpha_{i j}\right)$ represents various mobility coefficients [3].

Note that the volume fractions add up to 1 :

$$
\phi_{n}+\phi_{b}+\phi_{s}=1
$$

for incompressible mixtures. This along with the Onsager reciprocal principle requires

$$
\sum_{j=1}^{3} \alpha_{i j}=0, \alpha_{i j}=\alpha_{j i}
$$

and

$$
g_{n b}+g_{s b}+g_{s}=0
$$

Then, we deduce that

$$
g_{s}=-\left(g_{n b}+g_{s b}\right) .
$$

From (2.3), the off-diagonal mobility coefficients can be obtained from the diagonal coefficients,

$$
\begin{aligned}
& \alpha_{12}=\frac{1}{2}\left(\alpha_{33}-\alpha_{11}-\alpha_{22}\right), \\
& \alpha_{13}=\frac{1}{2}\left(\alpha_{22}-\alpha_{11}-\alpha_{33}\right), \\
& \alpha_{23}=\frac{1}{2}\left(\alpha_{11}-\alpha_{22}-\alpha_{33}\right) .
\end{aligned}
$$

Hence, for any given incompressible multicomponent fluid system, we only need to specify the principal mobility coefficients along the diagonal of the matrix $\left(\alpha_{i j}\right)$.

As a result, the system for the volume fractions is a system of modified or singular Cahn-Hilliard equations $[4,5]$ with reaction terms

$$
\begin{aligned}
\frac{\partial \phi_{n}}{\partial t}+\nabla \cdot\left(\phi_{n} \mathbf{v}\right)= & \nabla \cdot\left[\alpha_{11} \nabla\left(\frac{\delta f}{\delta \phi_{n}}-\frac{1}{2}\left(\frac{\delta f}{\delta \phi_{b}}+\frac{\delta f}{\delta \phi_{s}}\right)\right)\right. \\
& \left.+\frac{1}{2}\left(\alpha_{22}-\alpha_{33}\right) \nabla\left(\frac{\delta f}{\delta \phi_{s}}-\frac{\delta f}{\delta \phi_{b}}\right)\right]+g_{n b}, \\
\frac{\partial \phi_{b}}{\partial t}+\nabla \cdot\left(\phi_{b} \mathbf{v}\right)= & \nabla \cdot\left[\alpha_{22} \nabla\left(\frac{\delta f}{\delta \phi_{b}}-\frac{1}{2}\left(\frac{\delta f}{\delta \phi_{n}}+\frac{\delta f}{\delta \phi_{s}}\right)\right)\right. \\
& \left.+\frac{1}{2}\left(\alpha_{11}-\alpha_{33}\right) \nabla\left(\frac{\delta f}{\delta \phi_{s}}-\frac{\delta f}{\delta \phi_{n}}\right)\right]+g_{s b}, \\
\frac{\partial \phi_{s}}{\partial t}+\nabla \cdot\left(\phi_{s} \mathbf{v}\right)= & \nabla \cdot\left[\alpha_{33} \nabla\left(\frac{\delta f}{\delta \phi_{s}}-\frac{1}{2}\left(\frac{\delta f}{\delta \phi_{b}}+\frac{\delta f}{\delta \phi_{n}}\right)\right)\right. \\
& \left.+\frac{1}{2}\left(\alpha_{22}-\alpha_{11}\right) \nabla\left(\frac{\delta f}{\delta \phi_{n}}-\frac{\delta f}{\delta \phi_{b}}\right)\right]+g_{s} .
\end{aligned}
$$

From the system of equations for the volume fractions, we can identify the velocity for each individual effective component by accounting for the excessive velocity. For instance, we identify the solvent velocity as

$$
\mathbf{v}_{s}=\mathbf{v}-\frac{1}{\phi_{s}}\left[\alpha_{33} \nabla\left(\frac{\delta f}{\delta \phi_{s}}-\frac{1}{2}\left(\frac{\delta f}{\delta \phi_{b}}+\frac{\delta f}{\delta \phi_{n}}\right)\right)+\frac{1}{2}\left(\alpha_{22}-\alpha_{11}\right) \nabla\left(\frac{\delta f}{\delta \phi_{n}}-\frac{\delta f}{\delta \phi_{b}}\right)\right] .
$$


Analogously, we can identify the velocity of the EPS and bacteria respectively as

$$
\begin{aligned}
& \mathbf{v}_{n}=\mathbf{v}-\frac{1}{\phi_{n}}\left[\alpha_{11} \nabla\left(\frac{\delta f}{\delta \phi_{n}}-\frac{1}{2}\left(\frac{\delta f}{\delta \phi_{b}}+\frac{\delta f}{\delta \phi_{s}}\right)\right)+\frac{1}{2}\left(\alpha_{22}-\alpha_{33}\right) \nabla\left(\frac{\delta f}{\delta \phi_{s}}-\frac{\delta f}{\delta \phi_{b}}\right)\right], \\
& \mathbf{v}_{b}=\mathbf{v}-\frac{1}{\phi_{b}}\left[\alpha_{22} \nabla\left(\frac{\delta f}{\delta \phi_{b}}-\frac{1}{2}\left(\frac{\delta f}{\delta \phi_{n}}+\frac{\delta f}{\delta \phi_{s}}\right)\right)+\frac{1}{2}\left(\alpha_{11}-\alpha_{33}\right) \nabla\left(\frac{\delta f}{\delta \phi_{s}}-\frac{\delta f}{\delta \phi_{n}}\right)\right] .
\end{aligned}
$$

It then follows that

$$
\mathbf{v}=\phi_{n} \mathbf{v}_{n}+\phi_{b} \mathbf{v}_{b}+\phi_{s} \mathbf{v}_{s}
$$

So, the bulk velocity is indeed a volume averaged velocity.

Acknowledging that the contribution to the velocity of a species vanishes when the species does not exist, we approximate the mobility coefficients as follows in the paper

$$
\begin{aligned}
& \alpha_{11}=\lambda_{1} \phi_{n}\left(1-\phi_{n}\right), \\
& \alpha_{22}=\lambda_{2} \phi_{b}\left(1-\phi_{b}\right), \\
& \alpha_{33}=\lambda_{3} \phi_{s}\left(1-\phi_{s}\right),
\end{aligned}
$$

where $\lambda_{1,2,3}$ are three constants. From (2.11), if we assume $\lambda_{1}=\lambda_{2}=\lambda_{3}=\lambda$, we arrive at

$$
\begin{aligned}
& \alpha_{22}-\alpha_{11}=\lambda \phi_{s}\left(\phi_{b}-\phi_{n}\right), \\
& \alpha_{22}-\alpha_{33}=\lambda \phi_{n}\left(\phi_{b}-\phi_{s}\right), \\
& \alpha_{11}-\alpha_{33}=\lambda \phi_{b}\left(\phi_{n}-\phi_{s}\right) .
\end{aligned}
$$

This choice of the coefficients in the mobility matrix yields zero flux for a component when its volume fraction is zero.

The velocity for each individual component is then given respectively by

$$
\begin{aligned}
& \mathbf{v}_{s}=\mathbf{v}-\lambda\left[\left(1-\phi_{s}\right) \nabla\left(\frac{\delta f}{\delta \phi_{s}}-\frac{1}{2}\left(\frac{\delta f}{\delta \phi_{b}}+\frac{\delta f}{\delta \phi_{n}}\right)\right)+\frac{1}{2}\left(\phi_{b}-\phi_{n}\right) \nabla\left(\frac{\delta f}{\delta \phi_{n}}-\frac{\delta f}{\delta \phi_{b}}\right)\right] . \\
& \mathbf{v}_{n}=\mathbf{v}-\lambda\left[\left(1-\phi_{n}\right) \nabla\left(\frac{\delta f}{\delta \phi_{n}}-\frac{1}{2}\left(\frac{\delta f}{\delta \phi_{b}}+\frac{\delta f}{\delta \phi_{s}}\right)\right)+\frac{1}{2}\left(\phi_{b}-\phi_{s}\right) \nabla\left(\frac{\delta f}{\delta \phi_{s}}-\frac{\delta f}{\delta \phi_{b}}\right)\right], \\
& \mathbf{v}_{b}=\mathbf{v}-\lambda\left[\left(1-\phi_{b}\right) \nabla\left(\frac{\delta f}{\delta \phi_{b}}-\frac{1}{2}\left(\frac{\delta f}{\delta \phi_{n}}+\frac{\delta f}{\delta \phi_{s}}\right)\right)+\frac{1}{2}\left(\phi_{n}-\phi_{s}\right) \nabla\left(\frac{\delta f}{\delta \phi_{s}}-\frac{\delta f}{\delta \phi_{n}}\right)\right] .
\end{aligned}
$$

The second term on the right hand side of each velocity is the excessive velocity for that effective component, which is present completely due to mixing.

2.2. Reactive kinetics of each component in the biofilm. We denote the concentration of bacteria, the drug, and the nutrient as $B, d$, and $c$, respectively. To derive the transport equation for the volume fractions, we need to sort out the relation among the unknowns $\phi_{n}, \phi_{b}, \phi_{s}, B, d, c$ using reactive dynamics. We note that the bacterial volume fraction $\phi_{b}$ is proportional to the concentration of the bacteria,

$$
\phi_{b}=s B
$$

where $s$ is a specific volume of the bacterium. Note that the polymer is the product of the bacteria and maybe the nutrient. So, its growth rate should be exclusively proportional to B and maybe the nutrient concentration c, i.e., when either B or c is zero, the growth of the EPS should cease. We also assume the EPS network may decay under the influence of the drug and the depletion of the nutrient, i.e., the decay 
rate due to the drug treatment should be proportional to the drug concentration, which either changes the material property of the EPS polymer to shrink its volume or simply dissolves the polymer into the solvent. Thus, the kinetics for the EPS network strand is given by

$$
\begin{aligned}
\phi_{n}^{\prime} & =\text { growthrate }(B, c)-\text { decayrate }(d)=g_{n b}=\mu_{0} B \frac{c}{K_{c}+c}-C_{1} \phi_{n} \frac{d}{K_{1}+d} \\
& =\frac{\mu_{0}}{s} \phi_{b} \frac{c}{K_{c}+c}-C_{1} \phi_{n} \frac{d}{K_{1}+d} .
\end{aligned}
$$

where $\mu_{0}$ and $C_{1}$ measures the growth rate and decay rate of the EPS strand with respect to the bacterial concentration and the EPS, respectively, $K_{c}$ and $K_{1}$ are constants in the Michaelis-Menton kinetics used in the reactive terms.

The bacterial growth depends on the nutrient and the drug. The kinetics is governed by

$$
\begin{aligned}
B^{\prime} & =\left[\text { growthrate }(c, B)-\text { decayrate }\left(d, B, \phi_{n}\right)\right] \\
& =\frac{C_{2} c}{\left(K_{2}+c\right)}\left(B-C_{B} B^{2}\right)-\frac{C_{3} d}{\left(K_{3}+d\right)} B,
\end{aligned}
$$

where $K_{2,3}$ are half saturation constants, $C_{2}$ is a growth rate for bacteria, $C_{3}$ is a decay rate for bacteria due to drugs, and $C_{B}$ is a decay parameter for bacteria in the logistic kinetics.

Multiplying (2.16) by s, we arrive at

$$
\phi_{b}^{\prime}=g_{s b}=\left[\frac{C_{2} c}{\left(K_{2}+c\right)}\left(1-\frac{C_{B}}{s} \phi_{b}\right)-\frac{C_{3} d}{\left(K_{3}+d\right)}\right] \phi_{b},
$$

The nutrient decay rate is proportional to the bacterial consumption,

$$
c^{\prime}=\text { decayrate }\left(\phi_{b}, c\right)=g_{c}=-\left(C_{4} B\right) \frac{c}{K_{4}+c}=-\left(C_{4} \phi_{b}\right) \frac{c}{s\left(K_{4}+c\right)},
$$

where $K_{4}$ is a saturation constant and $C_{4}$ parametrizes the decay rate of the nutrient.

The drug concentration depends on the bacterial concentration as well as the EPS polymer concentration. It is consumed by bacteria and diluted by the EPS polymer via chemical reactions. Both the presence of bacteria and the EPS reduces the concentration of the drug.

$$
d^{\prime}=\text { decayrate }\left(B, \phi_{n}\right)=g_{d}=-\left(C_{5} B+C_{6} \phi_{n}\right) \frac{d}{d_{0}+d}=-\left(\frac{C_{5}}{s} \phi_{b}+C_{6} \phi_{n}\right) \frac{d}{d_{0}+d},(2.1
$$

where $d_{0}$ is a half saturation concentration, $C_{5}$ is the decay rate of the drug due to the bacteria-drug interaction while $C_{6}$ is that due to the EPS-drug interaction.

Owing to the incompressibility $\left(\phi_{n}+\phi_{b}+\phi_{s}=1\right)$,

$$
\phi_{s}^{\prime}=-\left(g_{n b}+g_{s b}\right)=g_{s} .
$$

It suffices to assume that the dead bacteria and dissolved EPS polymers are converted into effective solvent in this model!

2.3. Mixing kinetics. Since the molecular weight of bacteria is normally two orders of magnitudes larger than that of the solvent molecules, we treat it as an effective polymer whose collective behavior is viscous. We use the Flory-Huggin's mixing theory to model the mixing phenomena among the EPS, bacteria and the 
effective solvent. We denote the mixing free energy density by $f$,

$$
\begin{aligned}
f= & k T\left[\frac{\gamma_{11}}{2}\left\|\nabla \phi_{n}\right\|^{2}+\frac{\gamma_{12}}{2}\left\|\nabla \phi_{b}\right\|^{2}+\frac{\gamma_{13}}{2}\left\|\nabla \phi_{s}\right\|^{2}\right. \\
& \left.+\gamma_{2}\left[\frac{\phi_{n}}{N_{p}} \ln \phi_{n}+\frac{\phi_{b}}{N_{b}} \ln \phi_{b}+\phi_{s} \ln \phi_{s}+\chi_{b n} \phi_{n} \phi_{b}+\chi_{s b} \phi_{s} \phi_{b}+\chi_{s n} \phi_{n} \phi_{s}\right]\right]
\end{aligned}
$$

where $\gamma_{11,12,13}$ and $\gamma_{2}$ measure the strength of the distortional and bulk mixing free energy, respectively, $N_{p}$ and $N_{b}$ denote the polymerization index of the EPS and bacteria, respectively, $\chi_{b n, s b, s n}$ are the mixing parameters for the EPS and bacteria, bacteria and solvent, and solvent and EPS, respectively. We remark that we use the simplest distortional conformational energy in this mixing free energy density for simplicity. Another choice for the distortional conformational energy can be

$$
\begin{array}{r}
k T\left[\frac{\gamma_{11}}{2}\left\|\phi_{b} \nabla \phi_{n}-\phi_{n} \nabla \phi_{b}\right\|^{2}+\frac{\gamma_{12}}{2}\left\|\phi_{s} \nabla \phi_{b}-\phi_{b} \nabla \phi_{s}\right\|^{2}\right. \\
\left.+\frac{\gamma_{13}}{2}\left\|\phi_{n} \nabla \phi_{s}-\phi_{s} \nabla \phi_{n}\right\|^{2}\right] .
\end{array}
$$

In this paper, we stick to the free energy density given in (2.21).

The variation of the free energy density with respect to each volume fraction is given respectively by

$$
\begin{aligned}
& \frac{\delta f}{\delta \phi_{n}}=-k T \gamma_{11} \Delta \phi_{n}+\gamma_{2} k T\left[\frac{1}{N_{p}}\left(\ln \left(\phi_{n}\right)+1\right)+\chi_{b n} \phi_{b}+\chi_{s n} \phi_{s}\right], \\
& \frac{\delta f}{\delta \phi_{b}}=-k T \gamma_{12} \Delta \phi_{b}+\gamma_{2} k T\left[\frac{1}{N_{b}}\left(\ln \left(\phi_{b}\right)+1\right)+\chi_{b n} \phi_{n}+\chi_{s b} \phi_{s}\right], \\
& \frac{\delta f}{\delta \phi_{s}}=-k T \gamma_{13} \Delta \phi_{s}+\gamma_{2} k T\left[\left(\ln \left(\phi_{s}\right)+1\right)+\chi_{s n} \phi_{n}+\chi_{s b} \phi_{b}\right] .
\end{aligned}
$$

2.4. Constitutive equations. We use the volume fraction of the eps and bacteria as the primary unknowns. The volume fraction of the solvent is therefore calculated from the incompressibility constraint. The mixing free energy in terms of the two unknowns is given by

$$
\begin{aligned}
f= & k T\left[\frac{\gamma_{11}}{2}\left\|\nabla \phi_{n}\right\|^{2}+\frac{\gamma_{12}}{2}\left\|\nabla \phi_{b}\right\|^{2}+\frac{\gamma_{13}}{2}\left\|\nabla\left(\phi_{n}+\phi_{b}\right)\right\|^{2}\right. \\
& +\gamma_{2}\left[\frac{\phi_{n}}{N_{p}} \ln \phi_{n}+\frac{\phi_{b}}{N_{b}} \ln \phi_{b}+\left(1-\phi_{n}-\phi_{b}\right) \ln \left(1-\phi_{n}-\phi_{b}\right)\right. \\
& \left.\left.+\chi_{b n} \phi_{n} \phi_{b}+\chi_{s b}\left(1-\phi_{b}-\phi_{n}\right) \phi_{b}+\chi_{s n} \phi_{n}\left(1-\phi_{n}-\phi_{b}\right)\right]\right],
\end{aligned}
$$

The variation of the free energy is given by

$$
\delta f=\left(\frac{\delta f}{\delta \phi_{n}}-\frac{\delta f}{\delta \phi_{s}}\right) \delta \phi_{n}+\left(\frac{\delta f}{\delta \phi_{b}}-\frac{\delta f}{\delta \phi_{s}}\right) \delta \phi_{b}
$$

Notice that

$$
\delta \phi_{n}=\frac{\partial \phi_{n}}{\partial t} \delta t=-\nabla \cdot\left(\mathbf{v} \phi_{n}\right) \delta t, \quad \delta \phi_{b}=\frac{\partial \phi_{b}}{\partial t} \delta t=-\nabla \cdot\left(\mathbf{v} \phi_{b}\right) \delta t .
$$

We denote the free energy associated with the mixing by

$$
\mathbf{A}=\int f d \mathbf{x}
$$


Its variation is given by

$$
\delta \mathbf{A}=\int \delta f d \mathbf{x}=\int \mathbf{v} \cdot\left[\phi_{n} \nabla\left(\frac{\delta f}{\delta \phi_{n}}-\frac{\delta f}{\delta \phi_{s}}\right)+\phi_{b} \nabla\left(\frac{\delta f}{\delta \phi_{b}}-\frac{\delta f}{\delta \phi_{s}}\right)\right] \delta t d \mathbf{x} .
$$

It then follows that

$$
\delta f=-\mathbf{v} \cdot \mathbf{F}_{e}
$$

which implies

$$
\begin{aligned}
\mathbf{F}_{e}= & -\phi_{n} \nabla\left(\frac{\delta f}{\delta \phi_{n}}-\frac{\delta f}{\delta \phi_{s}}\right)-\phi_{b} \nabla\left(\frac{\delta f}{\delta \phi_{b}}-\frac{\delta f}{\delta \phi_{s}}\right) \\
= & -\gamma_{13} k_{B} T \nabla \cdot\left(\nabla \phi_{n} \nabla \phi_{b}+\nabla \phi_{b} \nabla \phi_{n}\right)-\nabla \cdot\left(\left(\gamma_{11}+\gamma_{13}\right) k_{B} T \nabla \phi_{n} \nabla \phi_{n}\right. \\
& \left.+\left(\gamma_{12}+\gamma_{13}\right) k_{B} T \nabla \phi_{b} \nabla \phi_{b}\right)+\nabla\left[\hat{f}\left(\phi_{n}, \phi_{b}\right)\right. \\
& +\gamma_{11} k_{B} T\left(\phi_{n} \Delta \phi_{n}+\frac{1}{2}\left\|\nabla \phi_{n}\right\|^{2}\right)+\gamma_{12} k_{B} T\left(\phi_{b} \Delta \phi_{b}+\frac{1}{2}\left\|\nabla \phi_{b}\right\|^{2}\right) \\
& \left.+\gamma_{13} k_{B} T\left(\phi_{n} \Delta \phi_{b}+\phi_{b} \Delta \phi_{n}+\nabla \phi_{n} \nabla \phi_{b}\right)\right],
\end{aligned}
$$

where $\hat{f}$ consists of the bulk terms and their first integrals in the mixing free energy.

The first part can be identified as a part of the stress due to the inhomogeneity of the volume fraction and the second part can be combined with the pressure. So, the elastic stress tensor due to mixing dynamics is given by

$$
\begin{aligned}
\tau_{e s}= & -k_{B} T\left[\left(\gamma_{11}+\gamma_{13}\right) \nabla \phi_{n} \nabla \phi_{n}+\left(\gamma_{12}+\gamma_{13}\right) \nabla \phi_{b} \nabla \phi_{b}\right. \\
& \left.+\gamma_{13}\left(\nabla \phi_{b} \nabla \phi_{n}+\nabla \phi_{n} \nabla \phi_{b}\right)\right] .
\end{aligned}
$$

We consider two models. In the first model, we assume the transport in the mixture is carried out by the velocity gradient of the average velocity. For the solvent, we assume it is viscous with the stress given by

$$
\tau_{s}=2 \eta_{s} \mathbf{D}
$$

where $\eta_{s}$ is the solvent viscosity. The bacteria is assumed extended Newtonian

$$
\tau_{b}=2 \eta_{b}(\mathbf{D}) \mathbf{D}
$$

where $\eta_{b}$ is a rate-of-strain dependent viscosity. The EPS polymer network strand is assumed viscoelastic obeying the Johnson-Segalman equation

$$
\frac{d}{d t} \tau_{n}-\mathbf{W} \cdot \tau_{n}+\tau_{n} \cdot \mathbf{W}-a\left[\mathbf{D} \cdot \tau_{n}+\tau_{n} \cdot \mathbf{D}\right]+\frac{1}{\lambda_{1}} \tau_{n}=2 \frac{\eta_{p}}{\lambda_{1}} \mathbf{D},
$$

where $\frac{d}{d t}=\frac{\partial}{\partial t}+\mathbf{v} \cdot \nabla$ is the material derivative, a is a rate parameter in $[-1,1], \eta_{p}$ is the polymeric viscosity and $\lambda_{1}$ is the relaxation time. The EPS network contribution to the stress is $a \phi_{n} \tau_{n}$. Since the velocity gradient used in this constitutive model is calculated from the average velocity, we name it the VA model. 
If we assume the transport of each component is carried out by their respective velocity, alternative constitutive equations for the stress tensors are proposed as follows

$$
\begin{aligned}
& \tau_{s}=2 \eta_{s} \mathbf{D}_{s}, \quad \tau_{b}=2 \eta_{b} \mathbf{D}_{b}, \\
& \frac{d}{d t} \tau_{n}-\mathbf{W}_{n} \cdot \tau_{n}+\tau_{n} \cdot \mathbf{W}_{n}-a\left[\mathbf{D}_{n} \cdot \tau_{n}+\tau_{n} \cdot \mathbf{D}_{n}\right]+\frac{1}{\lambda_{1}} \tau_{n}=2 \frac{\eta_{p}}{\lambda_{1}} \mathbf{D}_{n},
\end{aligned}
$$

where $\mathbf{D}_{s}, \mathbf{D}_{b}, \mathbf{D}_{n}$ are the rate of strain tensors calculated using the respective velocity field $\mathbf{v}_{s}, \mathbf{v}_{b}, \mathbf{v}_{n}, \mathbf{W}_{n}$ is the vorticity tensor calculated from $\mathbf{v}_{n}$ and $\frac{d}{d t}=$ $\frac{\partial}{\partial t}+\mathbf{v}_{n} \cdot \nabla$ is the material derivative for the EPS strand. We refer to this constitutive model the VN model.

2.5. Transport of the nutrient and drugs. The transport of the nutrient is carried out by the solvent velocity $\mathbf{v}_{s}$ :

$$
\frac{\partial}{\partial t}\left(\phi_{s} c\right)+\nabla \cdot\left(c \mathbf{v}_{s} \phi_{s}-D_{s} \phi_{s} \nabla c\right)=g_{c}
$$

where $D_{s}$ is the diffusion coefficient for the nutrient. Analogously, the drug is assumed to be transported by the solvent velocity as well

$$
\frac{\partial}{\partial t}\left(\phi_{s} d\right)+\nabla \cdot\left(d \mathbf{v}_{s} \phi_{s}-D_{d} \phi_{s} \nabla d\right)=g_{d},
$$

where $D_{d}$ is the diffusion coefficient for the drug.

2.6. Summary of the governing system of equations. In summary, the governing system of equations for the biofilm-solvent mixture consists of the following equations.

$$
\begin{aligned}
& \frac{\partial \phi_{n}}{\partial t}+\nabla \cdot\left(\phi_{n} \mathbf{v}\right)= \nabla \cdot\left[\alpha_{11} \nabla\left(\frac{\delta f}{\delta \phi_{n}}-\frac{1}{2}\left(\frac{\delta f}{\delta \phi_{b}}+\frac{\delta f}{\delta \phi_{s}}\right)\right)\right. \\
&\left.+\frac{1}{2}\left(\alpha_{22}-\alpha_{33}\right) \nabla\left(\frac{\delta f}{\delta \phi_{s}}-\frac{\delta f}{\delta \phi_{b}}\right)\right]+g_{n b} \\
& \frac{\partial \phi_{b}}{\partial t}+\nabla \cdot\left(\phi_{b} \mathbf{v}\right)= \nabla \cdot\left[\alpha_{22} \nabla\left(\frac{\delta f}{\delta \phi_{b}}-\frac{1}{2}\left(\frac{\delta f}{\delta \phi_{n}}+\frac{\delta f}{\delta \phi_{s}}\right)\right)+\right. \\
&\left.\frac{1}{2}\left(\alpha_{11}-\alpha_{33}\right) \nabla\left(\frac{\delta f}{\delta \phi_{s}}-\frac{\delta f}{\delta \phi_{n}}\right)\right]+g_{s b} \\
& \frac{\partial \phi_{s}}{\partial t}+\nabla \cdot\left(\phi_{s} \mathbf{v}\right)= \nabla \cdot\left[\alpha_{33} \nabla\left(\frac{\delta f}{\delta \phi_{s}}-\frac{1}{2}\left(\frac{\delta f}{\delta \phi_{b}}+\frac{\delta f}{\delta \phi_{n}}\right)\right)+\right. \\
&\left.\frac{1}{2}\left(\alpha_{22}-\alpha_{11}\right) \nabla\left(\frac{\delta f}{\delta \phi_{n}}-\frac{\delta f}{\delta \phi_{b}}\right)\right]+g_{s}, \\
& g_{n b}=\frac{\mu_{0}}{s} \phi_{b} \frac{c}{K_{c}+c}- {\left[C_{1} \phi_{n} \frac{d}{K_{1}+d}\right], } \\
& g_{s b}=\left[\frac{C_{2} c}{\left(K_{2}+c\right)}\left(1-\frac{C_{B}}{s} \phi_{b}\right)-\frac{C_{3} d}{\left(K_{3}+d\right)}\right] \phi_{b} \\
& g_{s}=-\left(g_{n b}+g_{s b}\right) .
\end{aligned}
$$

The velocity for each component is given respectively by 


$$
\begin{aligned}
& \mathbf{v}_{s}=\mathbf{v}-\lambda\left[\left(1-\phi_{s}\right) \nabla\left(\frac{\delta f}{\delta \phi_{s}}-\frac{1}{2}\left(\frac{\delta f}{\delta \phi_{b}}+\frac{\delta f}{\delta \phi_{n}}\right)\right)+\frac{1}{2}\left(\phi_{b}-\phi_{n}\right) \nabla\left(\frac{\delta f}{\delta \phi_{n}}-\frac{\delta f}{\delta \phi_{b}}\right)\right] \\
& \mathbf{v}_{n}=\mathbf{v}-\lambda\left[\left(1-\phi_{n}\right) \nabla\left(\frac{\delta f}{\delta \phi_{n}}-\frac{1}{2}\left(\frac{\delta f}{\delta \phi_{b}}+\frac{\delta f}{\delta \phi_{s}}\right)\right)+\frac{1}{2}\left(\phi_{b}-\phi_{s}\right) \nabla\left(\frac{\delta f}{\delta \phi_{s}}-\frac{\delta f}{\delta \phi_{b}}\right)\right] \\
& \mathbf{v}_{b}=\mathbf{v}-\lambda\left[\left(1-\phi_{b}\right) \nabla\left(\frac{\delta f}{\delta \phi_{b}}-\frac{1}{2}\left(\frac{\delta f}{\delta \phi_{n}}+\frac{\delta f}{\delta \phi_{s}}\right)\right)+\frac{1}{2}\left(\phi_{n}-\phi_{s}\right) \nabla\left(\frac{\delta f}{\delta \phi_{s}}-\frac{\delta f}{\delta \phi_{n}}\right)\right] .
\end{aligned}
$$

The transport equation for the nutrient and drug concentration are given respec-

tively by

$$
\begin{aligned}
& \frac{\partial\left(c \phi_{s}\right)}{\partial t}+\nabla \cdot\left(\mathbf{v}_{s} c \phi_{s}\right)=\nabla \cdot \phi_{s} D_{s} \nabla c-\left(C_{4} \phi_{b}\right) \frac{c}{s\left(K_{4}+c\right)} \\
& \frac{\partial\left(d \phi_{s}\right)}{\partial t}+\nabla \cdot\left(\mathbf{v}_{s} d \phi_{s}\right)=\nabla \cdot \phi_{s} D_{d} \nabla d-\left(\frac{C_{5}}{s} \phi_{b}+C_{6} \phi_{n}\right) d .
\end{aligned}
$$

The continuity equation and the momentum equation for the mixture are given respectively by

$$
\begin{aligned}
\nabla \cdot(\mathbf{v})= & 0 \\
\rho \frac{d \mathbf{v}}{d t}= & \nabla \cdot\left(a \phi_{n} \tau_{n}+\phi_{b} \tau_{b}+\phi_{s} \tau_{s}\right)-\nabla \cdot\left[p \mathbf{I}+k_{B} T\left(\left(\gamma_{11}+\gamma_{13}\right) \nabla \phi_{n} \nabla \phi_{n}\right.\right. \\
& \left.\left.+\left(\gamma_{12}+\gamma_{13}\right) \nabla \phi_{b} \nabla \phi_{b}+\gamma_{13}\left(\nabla \phi_{n} \nabla \phi_{b}+\nabla \phi_{b} \nabla \phi_{n}\right)\right)\right]
\end{aligned}
$$

where $\rho=\phi_{s} \rho_{s}+\phi_{b} \rho_{b}+\phi_{n} \rho_{n}$ is the density of the mixture. The stress constitutive equations are given by eq. (2.34) or (2.35). We remark that the elastic stress constitutive equation is valid only within the domain where $\phi_{n} \neq 0$. Hence, it is zero in the pure solvent region.

3. Nondimensionalization. We use a characteristic time scale $t_{0}$, a characteristic length scale $h$, a characteristic nutrient concentration $c_{0}$ and drug concentration $d_{0}$ to nondimensionalize the variables

$$
\tilde{t}=\frac{t}{t_{0}}, \tilde{\mathbf{x}}=\frac{\mathbf{x}}{h}, \tilde{\mathbf{v}}=\frac{\mathbf{v} t_{0}}{h}, \tilde{p}=\frac{p h^{2}}{f_{0}}, \tilde{\tau}=\frac{\tau h^{2}}{f_{0}}, \tilde{c}=\frac{c}{c_{0}}, \tilde{d}=\frac{d}{d_{0}},
$$

where $f_{0}$ is a characteristic force scale. The following dimensionless parameters arise

$$
\begin{aligned}
& \Lambda=\frac{\lambda t_{0} f_{0}}{h^{4}}, \Gamma_{1 j}=\frac{\gamma_{1 j} k_{B} T}{f_{0}}, j=1,2,3, \Gamma_{2}=\frac{\gamma_{2} k_{B} T h^{2}}{f_{0}}, R e_{s}=\frac{f_{0} t_{0}}{\eta_{s} h^{2}}, R e_{b}=\frac{f_{0} t_{0}}{\eta_{b} h^{2}} \\
& R e_{p}=\frac{f_{0} t_{0}}{\eta_{p} h^{2}}, \tilde{D}_{s}=\frac{D_{s} t_{0}}{h^{2}}, \tilde{D}_{d}=\frac{D_{d} t_{0}}{h^{2}}, B i=\frac{\rho_{0} h^{4}}{f_{0} t_{0}^{2}}, \tilde{\rho}=B_{i}\left(\phi_{s} \frac{\rho_{s}}{\rho_{0}}+\phi_{n} \frac{\rho_{n}}{\rho_{0}}\right) \\
& \tilde{\mu}_{0}=\frac{\mu_{0} t_{0}}{s}, \tilde{K}_{c}=\frac{K_{c}}{c_{0}}, \tilde{K}_{i}=\frac{K_{i}}{c_{0}}, i=1, \cdots, 3, \Lambda_{1}=\frac{\lambda_{1}}{t_{0}}, \tilde{C}_{1}=C_{1} t_{0}, C_{2}=C_{2} t_{0} \\
& \tilde{C}_{3}=C_{3} t_{0}, \tilde{C}_{4}=\frac{C_{4} t_{0}}{s}, \tilde{C}_{5}=\frac{C_{5} t_{0}}{s}, \tilde{C}_{6}=C_{6} t_{0}, \tilde{C}_{B}=\frac{C_{B}}{s} .
\end{aligned}
$$

where $\tilde{\rho}_{0}$ is an average density, $R e_{s}, R e_{b}$ and $R e_{p}$ are the Reynolds number for the solvent, the bacterium and the EPS, respectively, $\Lambda_{1}$ is the Deborah number for the EPS. 
For simplicity,we drop the ${ }^{\sim}$ on the dimensionless variables and the parameters. The system of governing equations in these dimensionless variables are given by

$$
\begin{aligned}
& \frac{\partial \phi_{n}}{\partial t}+\nabla \cdot\left(\phi_{n} \mathbf{v}\right)= \nabla \cdot\left[\Lambda \phi _ { n } \left(\left(1-\phi_{n}\right) \nabla\left(\frac{\delta f}{\delta \phi_{n}}-\frac{1}{2}\left(\frac{\delta f}{\delta \phi_{b}}+\frac{\delta f}{\delta \phi_{s}}\right)\right)\right.\right. \\
&\left.\left.+\frac{1}{2}\left(\phi_{b}-\phi_{s}\right) \nabla\left(\frac{\delta f}{\delta \phi_{s}}-\frac{\delta f}{\delta \phi_{b}}\right)\right)\right]+g_{n b}, \\
& \frac{\partial \phi_{b}}{\partial t}+\nabla \cdot\left(\phi_{b} \mathbf{v}\right)=\nabla \cdot\left[\Lambda \phi _ { b } \left(\left(1-\phi_{b}\right) \nabla\left(\frac{\delta f}{\delta \phi_{b}}-\frac{1}{2}\left(\frac{\delta f}{\delta \phi_{n}}+\frac{\delta f}{\delta \phi_{s}}\right)\right)\right.\right. \\
&\left.+\frac{1}{2}\left(\phi_{n}-\phi_{s}\right) \nabla\left(\frac{\delta f}{\delta \phi_{s}}-\frac{\delta f}{\delta \phi_{n}}\right)\right]+g_{s b},
\end{aligned}
$$

where $\rho=\phi_{s} \rho_{s}+\phi_{b} \rho_{b}+\phi_{n} \rho_{n}$. The constitutive equations for the VN model are given by

$$
\begin{array}{r}
\Lambda_{1}\left[\frac{\partial}{\partial t} \tau_{\mathbf{n}}+\mathbf{v}_{n} \cdot \nabla\left(\tau_{\mathbf{n}}\right)-\mathbf{W}_{n} \cdot \tau_{\mathbf{n}}+\tau_{\mathbf{n}} \cdot \mathbf{W}_{n}-a\left[\mathbf{D}_{n} \cdot \tau_{\mathbf{n}}+\tau_{\mathbf{n}} \cdot \mathbf{D}_{n}\right]\right] \\
+\tau_{n}=\frac{2}{R e_{p}} \mathbf{D}_{n},
\end{array}
$$

The mixing free energy density is now given by

$$
\begin{aligned}
f= & \frac{\Gamma_{11}}{2}\left\|\nabla \phi_{n}\right\|^{2}+\frac{\Gamma_{12}}{2}\left\|\nabla \phi_{b}\right\|^{2}+\frac{\Gamma_{13}}{2}\left\|\nabla \phi_{s}\right\|^{2} \\
& +\Gamma_{2}\left[\frac{\phi_{n}}{N_{p}} \ln \phi_{n}+\frac{\phi_{b}}{N_{b}} \ln \phi_{b}+\phi_{s} \ln \phi_{s}+\chi_{b n} \phi_{n} \phi_{b}+\chi_{s b} \phi_{s} \phi_{b}+\chi_{s n} \phi_{n} \phi_{s}\right] .
\end{aligned}
$$

The no-flux boundary conditions are derived from the transport equation for the volume fractions

$$
\begin{aligned}
& \mathbf{n} \cdot\left[\phi_{n}\left(\left(1-\phi_{n}\right) \nabla\left(\frac{\delta f}{\delta \phi_{n}}-\frac{1}{2}\left(\frac{\delta f}{\delta \phi_{b}}+\frac{\delta f}{\delta \phi_{s}}\right)\right)+\frac{1}{2}\left(\phi_{b}-\phi_{s}\right) \nabla\left(\frac{\delta f}{\delta \phi_{s}}-\frac{\delta f}{\delta \phi_{b}}\right)\right)\right]=0, \\
& \mathbf{n} \cdot\left[\phi_{b}\left(\left(1-\phi_{b}\right) \nabla\left(\frac{\delta f}{\delta \phi_{b}}-\frac{1}{2}\left(\frac{\delta f}{\delta \phi_{n}}+\frac{\delta f}{\delta \phi_{s}}\right)\right)+\frac{1}{2}\left(\phi_{n}-\phi_{s}\right) \nabla\left(\frac{\delta f}{\delta \phi_{s}}-\frac{\delta f}{\delta \phi_{n}}\right)\right]=0,\right.
\end{aligned}
$$

where $\mathbf{n}$ is the unit external normal of the boundary. In addition, we impose

$$
\mathbf{v}=\mathbf{0}, \mathbf{n} \cdot \nabla \phi_{n}=0, \mathbf{n} \cdot \nabla \phi_{b}=0 .
$$


Along most part of the boundary,

$$
\mathbf{n} \cdot \nabla c=0, \mathbf{n} \cdot \nabla d=0 .
$$

However, if we inject the drug along a certain part of the boundary, we impose the Dirichlet boundary condition along that part of the boundary

$$
d=d_{0} .
$$

Analogously, if the nutrient is fed along a certain part of the boundary, we impose

$$
c=c_{0}
$$

along that part of the boundary.

We denote the bulk free energy density as

$$
\hat{f}=\Gamma_{2}\left[\frac{\phi_{n}}{N_{p}} \ln \phi_{n}+\frac{\phi_{b}}{N_{b}} \ln \phi_{b}+\phi_{s} \ln \phi_{s}+\chi_{b n} \phi_{n} \phi_{b}+\chi_{s b} \phi_{s} \phi_{b}+\chi_{s n} \phi_{n} \phi_{s}\right] .
$$

In practice, we regularize the bulk free energy by the following in the case of $\phi_{n}=0$ and/or $\phi_{b}=0$ :

$$
\begin{aligned}
& \hat{f}=\Gamma_{2}\left[\frac{\phi_{n}+\Delta \phi_{n}}{N_{p}} \ln \left(\phi_{n}+\Delta \phi_{n}\right)+\frac{\phi_{b}+\Delta \phi_{b}}{N_{b}} \ln \left(\phi_{b}+\Delta \phi_{b}\right)+\phi_{s} \ln \phi_{s}+\right. \\
& \left.\chi_{b n} \phi_{n} \phi_{b}+\chi_{s b} \phi_{s} \phi_{b}+\chi_{s n} \phi_{n} \phi_{s}\right],
\end{aligned}
$$

where $\Delta \phi_{n}$ and $\Delta \phi_{b}$ are two small positive quantities. Note that we don't need to regularize $\phi_{s}$ since we always assume $\phi_{s}>0$ in the biofilm mixture.

It is worth noting here that our choice of characteristic timescale $t_{0}$ depends upon the physical phenomenon that we are interested in modeling, and has serious repercussions on the numerical stability of the scheme used to solve the equation. In fact, we could identify fast, slow, and intermediate timescales in the problem and propose some simplifications that arise in the case of very fast or very slow timescales.

Below, in figure 1, we plot the contour of the bulk mixing free energy function in the space of $\phi_{n}$ and $\phi_{b}$ for several choices of the model parameters. Since $\phi_{s}=$ $1-\phi_{b}-\phi_{n}$, we only show it for $\phi_{b}+\phi_{n} \leq 1$. The most pertinent feature shown in figure 1 is the valleys and wells, or local minima of the bulk energy function, and the dependence of the locations and depths of these minima with respect to the parameters. Notice that it is possible to have a local minimum, or even two local minima of the bulk mixing energy depending upon choices of mixing parameters and polymerization indices.

The emergence of the peaks and valleys depends non-trivially on the choice of model parameters. Our observations confirm that, for fixed polymerization indices, picking the mixing variable $\chi_{b n}$ to be zero will give rise to a single well potential. As this parameter is increased, the single well will gradually spread out, forming two wells that tend to move toward the $\phi_{n}$ and $\phi_{b}$ axes. This makes sense from a physical perspective, because the $\chi_{i j}$ terms are tied to the difference between the external enthalpy changes and the average of the internal entropy changes (i.e. expense of mixing between the two phases versus internal mixing). When $\chi_{b n}$ is zero, then the bulk mixing energy is minimized when $\phi_{n}$ and $\phi_{b}$ are equal because it is more expensive to interact internally. Likewise, when $\chi_{b n}$ is increased, it is cheaper to interact internally, and so the bulk mixing energy will be minimized by decreasing either phase and increasing the other.

The other mixing parameters also affect the surface, skewing the locations and depth of the wells to one side or another. For instance, if $\chi_{s n}$ is smaller than $\chi_{s b}$, 
the well nearest the $\phi_{b}$ axis will grow, and that nearest the $\phi_{n}$ axis will shrink. This means that the magnitude of the mixing energy associated with $\phi_{b}$ is larger, which makes sense since the mixing coefficient of the EPS network is lower. Likewise, if $\chi_{s b}$ is smaller than $\chi_{s n}$, then the opposite effect occurs. If we choose $\chi_{s b}=\chi_{s n}$, then the two wells are equally present. All of these results are illustrated in Figure 1. In Figure 1, the parameters aren't necessarily picked to reflect realistic parameters for biofilms, so in Figure 2, we plot a range of parameters that will be used for our biofilm model.

As demonstrated by Zhang et. al. [28], the surface of the bulk free energy determines the behavior of the solution, including the linear stability. In their case, the concavity of the bulk free energy function (viewed as a function of $\phi_{n}$ ) determined the behavior of the solution near steady state solutions. In this case, there are two phase variables, the natural extension is to analyze the eigenvalues of the Hessian matrix of the free energy to infer concavity. In particular, we care about the signs of the eigenvalues of the Hessian matrix. This eigenanalysis is consistent with the linear perturbation analysis for identifying stable and unstable long wave regimes (which will be formalized in a later section), with the negative eigenvalues corresponding to stable modes, and positive eigenvalues corresponding to unstable modes of the perturbation solution. Three of the regimes are shown in Figure 3.

It should be noted here that the values used to generate the graphs above are not necessarily specific to a biofilm model, but were chosen to show the types of behavior that can be exhibited from a general multi-fluid mixture. We will examine this behavior in the neighborhood of reasonable biofilm model parameters later.

4. Growth mechanisms of biofilms near constant steady-states. The system of equations admits two families of constant solutions:

$$
\begin{aligned}
& \phi_{n}=\phi_{n}^{0}, \phi_{b}=\phi_{b}^{0}, c=d=0, \mathbf{v}=\mathbf{v}_{0}, \text { and } \\
& \phi_{n}=0, \phi_{b}=0, c=c_{0}, d=d_{0}, \mathbf{v}=\mathbf{v}_{0},
\end{aligned}
$$

where $0 \leq \phi_{n}^{0}, \phi_{b}^{0} \leq 1$ are arbitrary constants satisfying the constraint (2.2) and $\mathbf{v}_{0}$ is an arbitrary constant vector. In the following, we investigate the linear stability of the constant steady states. Without loss of generality, we set $\mathbf{v}_{0}=0$ in the following analysis. Note that the linearized equations for the volume fractions decouple from the rest of the governing system of equations. We thus study the linear stability of this set of equations separately from the rest of the equations.

4.1. Linearized stability for equilibrium 1. We first consider the linearized system corresponding to the constant steady state(s) $\phi_{n}^{0}$ and $\phi_{b}^{0}$ with $c=0$ and $d=0$, which we call equilibrium 1 . The decoupled linearized system for the volume fractions is given by:

$$
\frac{\partial}{\partial t}\left(\begin{array}{c}
\delta \phi_{n} \\
\delta \phi_{b}
\end{array}\right)=\left(\begin{array}{cc}
\left.\frac{\partial}{\partial \phi_{n}} F\right|_{\phi_{n}^{0}, \phi_{b}^{0}} & \left.\frac{\partial}{\partial \phi_{b}} F\right|_{\phi_{n}^{0}, \phi_{b}^{0}} \\
\left.\frac{\partial}{\partial \phi_{n}} G\right|_{\phi_{n}^{0}, \phi_{b}^{0}} & \left.\frac{\partial}{\partial \phi_{b}} G\right|_{\phi_{n}^{0}, \phi_{b}^{0}}
\end{array}\right)\left(\begin{array}{c}
\delta \phi_{n} \\
\delta \phi_{b}
\end{array}\right)+O\left(\delta \phi_{n}^{2}, \delta \phi_{b}^{2}\right),
$$

where $F$ and $G$ are the right hand sides of equations (3.3-1) and (3.3-2) respectively, with $\phi_{s}$ replaced by $1-\left(\phi_{n}+\phi_{b}\right)$.

We define,

$$
A=\left(\begin{array}{cc}
\left.\frac{\partial}{\partial \phi_{n}} F\right|_{\phi_{n}^{0}, \phi_{b}^{0}} & \left.\frac{\partial}{\partial \phi_{b}} F\right|_{\phi_{n}^{0}, \phi_{b}^{0}} \\
\left.\frac{\partial}{\partial \phi_{n}} G\right|_{\phi_{n}^{0}, \phi_{b}^{0}} & \left.\frac{\partial}{\partial \phi_{b}} G\right|_{\phi_{n}^{0}, \phi_{b}^{0}}
\end{array}\right),
$$


with a similar matrix for the coupled stress/velocity equations and the nutrient/drug equations which will be explored shortly. The linearized eigenfunctions associated with the phase variables and the nutrient and drug concentration $(\phi$ and $c, d)$ is $\cos (\beta y)$, while that associated with the velocity components is $\sin (\beta y)$, where $\beta=$ $m \pi, m=1, \ldots, \infty$. For an eigenfunction solution of this form, the components of $A$ are,

$$
\begin{aligned}
a_{11}= & -\Lambda \phi_{n}^{0}\left[\beta^{4}\left(\left(\Gamma_{11}+\Gamma_{13}\right)\left(1-\phi_{n}^{0}\right)-\Gamma_{13} \phi_{b}^{0}\right)+\right. \\
& \left.\beta^{2} \Gamma_{2}\left(\frac{1-\phi_{n}^{0}}{N_{p} \phi_{n}^{0}}-2\left(1-\phi_{n}^{0}\right) \chi_{s n}+1+\phi_{b}^{0}\left(\chi_{s n}-\chi_{b n}+\chi_{s b}\right)\right)\right] \\
a_{22}= & -\Lambda \phi_{b}^{0}\left[\beta^{4}\left(\left(\Gamma_{12}+\Gamma_{13}\right)\left(1-\phi_{b}^{0}\right)-\Gamma_{13} \phi_{n}^{0}\right)+\right. \\
& \left.\beta^{2} \Gamma_{2}\left(\frac{1-\phi_{b}^{0}}{N_{b} \phi_{b}^{0}}-2\left(1-\phi_{b}^{0}\right) \chi_{s b}+1+\phi_{n}^{0}\left(\chi_{s n}-\chi_{b n}+\chi_{s b}\right)\right)\right] \\
a_{12}= & -\Lambda \phi_{n}^{0}\left[\beta^{4}\left(\Gamma_{13}\left(1-\phi_{n}^{0}\right)-\left(\Gamma_{12}+\Gamma_{13}\right) \phi_{b}^{0}\right)+\right. \\
& \left.\beta^{2} \Gamma_{2}\left(\frac{-1}{N_{b}}+2 \phi_{b}^{0} \chi_{s b}+1+\left(1-\phi_{n}^{0}\right)\left(\chi_{b n}-\chi_{s n}+\chi_{s b}\right)\right)\right] \\
a_{21}= & -\Lambda \phi_{b}^{0}\left[\beta^{4}\left(\Gamma_{13}\left(1-\phi_{b}^{0}\right)-\left(\Gamma_{11}+\Gamma_{13}\right) \phi_{n}^{0}\right)+\right. \\
& \left.\beta^{2} \Gamma_{2}\left(\frac{-1}{N_{p}}+2 \phi_{n}^{0} \chi_{s n}+1+\left(1-\phi_{b}^{0}\right)\left(\chi_{b n}-\chi_{s b}+\chi_{s n}\right)\right)\right] .
\end{aligned}
$$

With the coefficients, one can easily compute the linearized growth rates,

$$
\sigma_{1}, \sigma_{2}=\frac{\operatorname{tr}(A) \pm \sqrt{\operatorname{tr}(A)^{2}-4 \operatorname{det}(A)}}{2} .
$$

Interestingly, if we consider the case $\phi_{n}=\phi_{n}^{0}$ and $\phi_{b}=\phi_{b}^{0}=0$, then $a_{22}=a_{21}=0$ and the only nonzero term governing the linearized growth rates (the eigenvalues of $A$ ) is the $a_{11}$ term:

$a_{11}=\Lambda \phi_{n}^{0} \beta^{2}\left(1-\phi_{n}^{0}\right)\left(-\left(\Gamma_{11}+\Gamma_{13}\right) \beta^{2}-\Gamma_{2}\left(\frac{1}{N_{p}\left(\phi_{n}^{0}+\Delta \phi_{n}\right)}+\frac{1}{1-\phi_{n}^{0}}-2 \chi_{s n}\right)\right)$,

which recovers the binary growth rates for the modified Cahn-Hilliard model obtained in [28].

In the regime of short wave lengths $|\beta|>>1$, the rates $\sigma_{1,2}$ are negative. However, they may be positive in the regime of long waves $(|\beta|<<1)$. In the long wave regime, the growth rates are given asymptotically by

$$
\sigma_{1,2} \approx \frac{\beta^{2} \Lambda \Gamma_{2}}{2}\left[-\operatorname{Tr}(R) \pm \sqrt{(\operatorname{Tr}(R))^{2}-4 \operatorname{Det}(R)}\right]
$$

where

$$
\begin{aligned}
\operatorname{Tr}(R)= & \phi_{b}+\phi_{n}+\frac{1-\phi_{b}}{N_{b}}+\frac{1-\phi_{n}}{N_{p}} \\
& -2\left[\left(1-\phi_{n}-\phi_{b}\right)\left(\phi_{n} \chi_{s n}+\phi_{b} \chi_{s b}\right)+\phi_{n} \phi_{b} \chi_{b n}\right], \\
\operatorname{Det}(R)= & \phi_{n} \phi_{b}\left[( 1 - \phi _ { n } - \phi _ { b } ) \left(4 \chi_{s n} \chi_{s b}-\mu^{2}-\frac{2 \chi_{s b}}{N_{p} \phi_{n}}\right.\right. \\
& \left.\left.-\frac{2 \chi_{s n}}{N_{b} \phi_{b}}+\frac{1}{N_{b} \phi_{b}}+\frac{1}{N_{p} \phi_{n}}\right)-2 \chi_{b n}\right] .
\end{aligned}
$$

Here $\mu=\chi_{s n}+\chi_{s b}-\chi_{b n}$. There are three scenarios for the growth rates.

- If $\operatorname{Tr}(R)<0$ and $\operatorname{Det}(R)>0$, there are two positive growth rates. 
- If $\operatorname{Det}(R)<0$, there is one positive growth rate in the long wave regime.

- If $\operatorname{Tr}(R)>0$ an $\mathrm{d} \operatorname{Det}(R)>0$, there is no positive growth rate.

In Figure 4, we plot the growth rates as functions of the wave number for each of the three scenarios. Note that the three possibilities discussed above correspond precisely to the earlier eigenanalysis of the Hessian matrix for the bulk free energy. Thus, there is a precise relationship between the curvature of the mixing free energy surface and the long wave stability of the steady state solution.

One of the pertinent features of the growth rates plotted above in Figure 4 is the emergence of unstable long wave numbers. As demonstrated, these unstable wave numbers can occur in both, one of, or neither of the eigenvalues, and thus a more detailed algebraic study of this phenomenon is warranted. Since all modes are stable at short wave regimes, we will refer to the transition from an unstable to a stable eigenmode in either wave number space or parameter space as the "cutoff parameter(s)" and will also try to find the maximum unstable wave number for either of the growth rates. Despite the desirability of a simple solution to the cut off parameter, the complexity of the structure of the various coefficients in the matrix $A$ has yielded solutions only by using CAS, namely Maple.

The solution for the cutoff parameter emits at most four non-zero solutions that are two sets of paired solutions (one solution for the cutoff parameter of each eigenvalue), and at least two trivial solutions. We note that unstable wavenumbers exist if and only if the above equation has positive solutions in parameter space, otherwise the system is universally stable for the constant solutions discussed above. The solution for the cutoff parameter is calculated using a CAS, and the exact form is suppressed here for readability.

We can plot the cutoff wavenumber i.e. the transition from a stable to an unstable wave number as a function of any of the parameters in the problem. Figure 5 plots the cutoff wave number as a function of the mixing variables. For fixed $\Gamma_{11}=$ $\Gamma_{12}=\Gamma_{13}$ we can give the cutoff wave number as a function of the ratio of $\Gamma_{2}$ to $\Gamma_{11}$ $\beta_{\text {cutoff }}=\left\{g_{1} / \sqrt{\Gamma_{11} / \Gamma_{2}}, g_{2} / \sqrt{\Gamma_{11} / \Gamma_{2}}\right\}$, where $g_{1}$ and $g_{2}$ are constants dependent on the model parameters, and is illustrated in Figure 5. The figures below are labeled to indicate wave numbers that are stable in both eigenvalues, unstable in a single eigenvalue, and unstable in both eigenvalues.

Again, it is worth noting that the values chosen above aren't necessarily specific to any biofilm model, but are chosen to demonstrate the emergence of the different categories of long wave instability present in this model. In fact, for values that we believe to be reasonable for biofilms (see table 1), the predominant behavior is of the second type of linearized solution, i.e. exactly one unstable eigenmode.

4.1.1. Viscous limit. The time scale for the EPS relaxation and the one for the biofilm overall growth are far apart. The former is normally in seconds and the latter in perhaps hours. When we are interested in the phenomenon in the biofilm growth time scale, the Deborah number $\left(\Lambda_{1}\right)$ is normally very small. When $\Lambda_{1} \ll 1$, the elastic stress constitutive equation reduces to the viscous limit. In this limit, the rest of the growth rates for the linearized system are given by:

$$
\begin{aligned}
& \sigma_{3}=-D_{s} \beta^{2} \\
& \sigma_{4}=-D_{d} \beta^{2} \\
& \sigma_{5,6}=-\frac{\beta^{2}}{\rho_{0}}\left(a \frac{\phi_{n}^{0}}{R e_{p}}+\frac{\phi_{b}^{0}}{R e_{b}}+\frac{1-\phi_{b}^{0}-\phi_{n}^{0}}{R e_{s}}\right),
\end{aligned}
$$


where $\sigma_{3}$ and $\sigma_{4}$ give the growth rate for the nutrient and drug concentration, respectively, and $\sigma_{5}$ and $\sigma_{6}$ give the growth rates for the non-zero velocity components $v_{x}$ and $v_{z}$ (in the viscous limit). Note the minus signs on the growth rates for the drug, nutrient, and velocity terms are all negative, indicating pure decay of these components.

4.1.2. Viscoelastic Growth rates. When the time scale in the phenomenon we are interested in is comparable to the EPS relaxation time, the system is in the viscoelastic regime. In this regime, the biofilm growth is negligible. Here, we continue our systematic approach by considering the VA model with a nonlinear constitutive equation for the elastic stress $\tau_{n}$. In this case, the velocity and the stress are coupled in two velocity components and two stress components in the linearized equation. These are $v_{x}, v_{z}, \tau_{x y}$, and $\tau_{x z}$. Again, we consider the matrix form of the linearized system and find the linearized growth rates for the coupled system, which are given as the eigenvalues of the matrix:

$$
B=\left(\begin{array}{cccc}
-\frac{\beta^{2}}{\rho_{0}}\left(\frac{\phi_{b}^{0}}{R e_{b}}+\frac{1-\phi_{n}^{0}-\phi_{b}^{0}}{R e_{s}}\right) & 0 & a i \phi_{n}^{0} \beta & 0 \\
0 & -\frac{\beta^{2}}{\rho_{0}}\left(\frac{\phi_{b}^{0}}{R e_{b}}+\frac{1-\phi_{n}^{0}-\phi_{b}^{0}}{R e_{s}}\right) & 0 & a i \phi_{n}^{0} \beta \\
i \beta / R e_{p} \Lambda_{1} & 0 & -1 / \Lambda_{1} & 0 \\
0 & i \beta / R e_{p} \Lambda_{1} & 0 & -1 / \Lambda_{1}
\end{array}\right) .
$$

The growth rates have two repeated eigenvalues given by

$$
\begin{aligned}
\sigma_{i, i+1, i+2, i+3}= & -\frac{1}{2}\left(\frac{1}{\Lambda_{1}}+\frac{\beta^{2} \phi_{b}^{0}}{\rho R e_{b}}+\frac{\beta^{2} \phi_{s}^{0}}{\rho R e_{s}}\right. \\
& \pm \sqrt{\left.\left(-\frac{1}{\Lambda_{1}}+\frac{\beta^{2} \phi_{b}^{0}}{\rho R e_{b}}+\frac{\beta^{2} \phi_{s}^{0}}{\rho R e_{s}}\right)^{2}-4 \frac{a \phi_{n}^{0} \beta^{2}}{R e_{p} \Lambda_{1}}\right)}
\end{aligned}
$$

All growth rates here are always negative, indicating linear decay of the modes. The uncoupled modes of the linearized stress tensor are decay rates given by

$$
\sigma_{i+4, i+5, i+6, i+7}=-\frac{1}{\Lambda_{1}} \text {. }
$$

The index for the growth rates starts at $i=5$. We remark that the VN-model growth rates for the coupled velocity/stress terms are the same as those just derived.

4.2. Linear stability analysis for equilibrium 2. For the second family of steady state solutions with a non-zero nutrient and drug concentration $c=c_{0}$, and $d=d_{0}$ the matrix $A$ for the linearized system becomes:

$$
A=\left(\begin{array}{cc}
a_{11}+m_{11} & a_{12}+m_{12} \\
a_{21} & a_{22}+m_{22}
\end{array}\right),
$$

with the various $m_{i j}$ s given by the partial derivatives of $g_{s b}$ and $g_{n b}$ with respect to $\phi_{b}$ and $\phi_{n}$, respectively,

$$
m_{11}=-\frac{C_{1} d_{0}}{K_{1}+d_{0}}, \quad m_{12}=\frac{\mu_{0} c_{0}}{K_{c}+c_{0}}, \quad m_{22}=\frac{C_{2} c_{0}}{K_{2}+c_{0}}-\frac{C_{3} d_{0}}{K_{3}+d} .
$$

Notice that only the diagonal entries of $A, m_{11}$ and $m_{22}$, contribute to the growth of $\phi_{n}$ and $\phi_{b}$ at the equilibrium $\phi_{n}^{0}=\phi_{b}^{0}=0$, and so the growth of the biofilm is given as: 


$$
\begin{aligned}
\sigma_{1} & =-\frac{C_{1} d_{0}}{K_{1}+d_{0}}, \\
\sigma_{2} & =\frac{C_{2} c_{0}}{K_{2}+c_{0}}-\frac{C_{3} d_{0}}{K_{3}+d} .
\end{aligned}
$$

Here, $\sigma_{1}$ is the growth rate of the EPS network and $\sigma_{2}$ is the growth rate of the bacteria population. Note that the EPS network is decaying, while the growth rate of the bacteria depends on the amount of nutrient versus the amount of drug present. The linearized growth rates for the evolution of the concentrations $c$ and $d$ are:

$$
\begin{aligned}
& \sigma_{3}=-D_{s} \beta^{2} \\
& \sigma_{4}=-D_{d} \beta^{2},
\end{aligned}
$$

which indicate pure decay for both the nutrient and drug concentrations. The only possible growth comes from the bacterial growth for this steady state solution.

The linearized growth rates for the constant velocity terms $v_{x}$ and $v_{z}$ in the viscous limit are given by:

$$
\sigma_{5,6}=-\frac{\beta^{2}}{\rho_{0}}\left(\frac{1}{R e_{s}}\right) .
$$

The growth rates for the elastic stress components are identical to the case of equilibrium 1 evaluated at equilibrium $\phi_{n}^{0}=\phi_{b}^{0}=0$. In the above analysis, the velocity term $\boldsymbol{v}$ is the average of the velocity components, which is the VA model. Of course, this analysis can be performed for the other model. In the VN model, the velocity and stress still are coupled with the phase variables, in which the velocity components $v_{n}, v_{b}$ and $v_{s}$, are given as the vector $\boldsymbol{v}$ minus excessive velocity terms. The linearization about equilibrium 2 gives a large block diagonal system where the blocks along the center contain the same eigenvalues (and thus, growth rates) for the velocity components as the VA model. The only difference between the two models, in the linearized limit, is the appearance of isolated off diagonal entries which don't affect the growth rates, but do affect the eigenvectors associated with these growth rates.

5. Transient 1-D solutions. We present numerical solutions for transient states of the ternary biofilm model. In this section, we will investigate the growth of a biofilm in 1-D: $y \in I=[0,1]$, governed by the equations given above. We consider a 1-D initial-boundary value problem with prescribed initial states:

$$
\phi_{n}(y, 0)=\phi_{n}^{0}(y), \phi_{b}(y, 0)=\phi_{b}^{0}(y), c(y, 0)=c_{0}(y), d(y, 0)=d_{0}(y),
$$

and boundary conditions given by (3.7-3.10). We feed in the nutrient and drug concentration at the upper boundary:

$$
\left.c\right|_{y=1}=c^{*},\left.\quad d\right|_{y=1}=d^{*}(t),
$$

which allows the possibility of adding the drug/disinfectant in doses in time. We assume the velocity at $y=0$ is $\mathbf{v}=0$ and $\mathbf{v}(y=1)=\left(v_{0}, 0,0\right)$, where $v_{0}$ is a shear speed. From the continuity equation, we have $v_{y}=0$. Therefore, $\mathbf{v}=$ $\left(v_{x}(y, t), 0, v_{z}(y, t)\right)$ for the biofilm homogeneous in $(x, z)$ direction. 
5.1. Numerical schemes. The numerical scheme used to study the dynamics of biofilm growth will be a finite difference scheme. We use uniform spatial and time step sizes denoted by $\Delta y$ and $\Delta t$ respectively. For the solutions at a time step $n-1$ and $n$, the polymer volume fraction at time step $n+1$ governed by the Cahn-Hillard equation is calculated by:

$$
\frac{\phi_{n}^{n+1}-\phi_{n}^{n}}{\Delta t}+\theta \Lambda \nabla \cdot \Phi_{n}^{n+1}=\bar{g}_{n}^{n+\theta}-(1-\theta) \Lambda \nabla \cdot \Phi_{n}^{n}-\overline{N L}_{n}^{n+\theta},
$$

where the semi-implicit and explicit terms are split (the nonlinear terms, including the natural log terms from the free energy and from the growth rate, are extrapolated). For readability, we've introduced symbols corresponding to the explicit and semi-implicit/extrapolated pieces:

$$
\begin{aligned}
\Phi_{n}^{n+1}= & \bar{\phi}_{n}^{n+\theta}\left(1-\bar{\phi}_{n}^{n+\theta}\right) \nabla_{y}\left[\left(-\Gamma_{11}-.5 \Gamma_{13}\right) \Delta \phi_{n}^{n+1}-.5\left(-\Gamma_{12}+\Gamma_{13}\right) \Delta \phi_{b}^{n+1}+\right. \\
& \left.+\Gamma_{2}\left(-1.5 \chi_{s n}-.5 \chi_{b n}+.5 \chi_{s b}\right) \phi_{n}^{n+1}+\Gamma_{2}\left(\chi_{b n}-\chi_{s n}\right) \phi_{b}^{n+1}\right]+ \\
& +\bar{\phi}_{n}^{n+\theta}\left(2 \bar{\phi}_{b}^{n+\theta}+\bar{\phi}_{n}^{n+\theta}-1\right) \nabla_{y}\left[.5 \Gamma_{13} \Delta \phi_{n}^{n+1}+.5\left(\Gamma_{12}+\Gamma_{13}\right) \Delta \phi_{b}^{n+1}\right. \\
& \left.+.5 \Gamma_{2}\left(\chi_{s n}-\chi_{b n}+\chi_{s b}\right) \phi_{n}^{n+1}+\Gamma_{2} \chi_{s b} \phi_{b}^{n+1}\right] \\
\overline{N L}^{n+1}= & \bar{\phi}_{n}^{n+\theta}\left(1-\bar{\phi}_{n}^{n+\theta}\right) \nabla_{y}\left(\frac{1}{N_{p}}\left(\ln \left(\bar{\phi}_{n}^{n+\theta}+\Delta \phi_{n}^{0}\right)+1\right)\right. \\
& \left.-\frac{1}{2 N_{b}}\left(\ln \left(\bar{\phi}_{b}^{n+\theta}+\Delta \phi_{b}\right)+1\right)+\ln \bar{\phi}_{s}^{n+\theta}\right) \\
& +\bar{\phi}_{n}^{n+\theta}\left(2 \bar{\phi}_{b}^{n+\theta}+\bar{\phi}_{n}^{n+\theta}-1\right) \nabla_{y}\left(-\frac{1}{N_{b}}\left(\ln \left(\bar{\phi}_{b}^{n+\theta}+\Delta \phi_{b}\right)+1\right)\right. \\
& \left.+\ln \bar{\phi}_{s}^{n+\theta}\right) .
\end{aligned}
$$

A similar update scheme is employed for the other phase variable $\phi_{b}$. Here the $\theta$ method is employed for $0 \leq \theta \leq 1$ and $\theta=1 / 2$ gives the semi-implicit, second order in time Crank-Nicholson algorithm. Here, all of the "bar" terms are extrapolated values at the intermediate timestep: $(\cdot)^{n+\theta}=(1+\theta)(\cdot)^{n}-\theta(\cdot)^{n-1}$. Update schemes are employed for the nutrient and disinfectant concentration. The update equation for the nutrient transport equations is:

$$
\begin{gathered}
\frac{1}{\Delta t}\left(\phi_{s}^{n+1} c^{n+1}-\phi_{s}^{n+1} c^{n}\right)-\theta \nabla \cdot\left(D_{s} \phi_{s}^{n+1} \nabla c^{n+1}\right)= \\
(1-\theta) \nabla \cdot\left(D_{s} \phi_{s}^{n} \nabla c^{n}\right)-\bar{g}_{c}^{n+\theta}-\nabla \cdot\left(\bar{c}^{n+\theta} \overline{\mathbf{v}}_{s}^{n+\theta} \bar{\phi}_{s}^{n+\theta}\right),
\end{gathered}
$$

where the $\phi_{s}^{n+1}$ is computed by updating the phase field equations using the above scheme prior to updating the concentration of nutrient substrates. An analogous scheme is used for computing the dynamics of the drug/disenfectant.

The update scheme for the nonzero velocity component $v_{x}$ is given by the semi implicit $\theta$ method as well:

$$
\rho \frac{v_{x}^{n+1}-v_{x}^{n}}{\Delta t}-\theta \frac{\partial}{\partial y}\left(\Psi^{n+1} \frac{\partial}{\partial y} v_{x}^{n+1}\right)=(1-\theta) \frac{\partial}{\partial y}\left(\Psi^{n} \frac{\partial}{\partial y} v_{x}^{n}\right)+\frac{\partial}{\partial y}\left(a \bar{\phi}_{n}^{n+\theta} \bar{\tau}_{x y}^{n+\theta}\right) .
$$

Here, $\Psi^{n}=\left(\frac{\phi_{b}^{n}}{R e_{b}}+\frac{\phi_{s}^{n}}{R e_{s}}\right)$, recalling that $\phi_{s}=1-\phi_{n}-\phi_{b}$. Note that the boundary conditions for the velocity are explicit at $y=0,1$. The scheme for $v_{z}$ is analogous. All the second order dissipative terms are discretized using central differences leading to overall second order accuracy in space. 
For the elastic stress equations, all six components of the elastic stress tensor satisfy a generic equation of the form:

$$
\frac{\partial \tau_{\alpha \beta}}{\partial t}+v_{y}^{e} \frac{\partial \tau}{\partial y}=F_{\alpha \beta}\left(\tau, \nabla \mathbf{v}_{n}\right), \alpha, \beta=1, \cdots, 3 .
$$

For the interior of the numerical domain, we update the elastic stress tensor using an upwind scheme:

$$
\begin{aligned}
\frac{\tau_{\alpha \beta, j}^{n+1}-\tau_{\alpha \beta, j}^{n}}{\Delta t}= & -\frac{1}{2 \Delta y}\left\{\left[1-\operatorname{sign}\left(v_{y, j+1 / 2}^{e, n}\right)\right] v_{y, j+1 / 2}^{e, n}\left(\tau_{\alpha, \beta, j+1}^{n}-\tau_{\alpha \beta, j}^{n}\right)\right. \\
& \left.+\left[1-\operatorname{sign}\left(v_{y, j-1 / 2}^{e, n}\right)\right] v_{y, j-1 / 2}^{e, n}\left(\tau_{\alpha \beta, j}^{n}-\tau_{\alpha \beta, j-1}^{n}\right)\right\}+F\left(\tau_{j}^{n}, \nabla \boldsymbol{v}_{n}^{n}\right),
\end{aligned}
$$

where the $F\left(\tau_{j}^{n}, \nabla \boldsymbol{v}_{n}^{n}\right)$ is given by the right hand side of the elastic stress tensor equations [28]. At the boundaries, $v_{y}^{e}=0$, and there are no boundary conditions for the elastic stress tensor, meaning $\tau$ satisfies an $\operatorname{ODE} \frac{\partial \tau}{\partial t}=F(\tau, \boldsymbol{v})$ at $y=0,1$. At the discrete time steps, we update $\tau$ at the boundaries by employing the following Runge-Kutta method.

$$
\tau^{n+1}=\tau^{n}+\frac{\Delta t}{6}\left(K_{1}+2 K_{2}+2 K_{3}+K_{4}\right)
$$

where,

$$
\begin{aligned}
& K_{1}=F\left(\tau^{n}, \nabla \boldsymbol{v}_{n}^{n}\right), \\
& K_{2}=F\left(\tau^{n}+\frac{\Delta t}{2} K_{1} \nabla\left[\frac{\boldsymbol{v}_{n}^{n}+\boldsymbol{v}_{n}^{n+1}}{2}\right]\right), \\
& K_{3}=F\left(\tau^{n}+\frac{\Delta t}{2} K_{2} \nabla\left[\frac{\boldsymbol{v}_{n}^{n}+\boldsymbol{v}_{n}^{n+1}}{2}\right]\right), \\
& K_{4}=F\left(\tau^{n}+\Delta t K_{3} \nabla \boldsymbol{v}_{n}^{n+1}\right) .
\end{aligned}
$$

The time stepping in this scheme for the elastic stress is confined by the sizes of the convective coefficients.

5.2. Numerical results and discussions. Before taking a look at several numerical results, it is necessary to examine the way the various parameters affect the evolution of biofilm mixing and biofilm growth.

5.2.1. Model parameters and their effects. First, we examine the mobility $\Lambda$ and the mixing parameters to chronicle their effects on biofilm dynamics and, in particular, the mixing between the various phases. The parameter $\Lambda$ is the dominating parameter in the dissipation of the various phases in the biofilm mixture, and controls the magnitude of dissipation. In the studies where the inert biofilm is presumed to be slowly growing, we suggest $\Lambda \sim 10^{-10}$, whereas if a rapid growing biofilm is modeled (one breaking up or spreading out over a region), we suggest a larger value of $\Lambda \sim 10^{-8}$. Typical parameter values used for the simulations are given in the table below. Figure 6 contains several figures which illustrate the effects of the key model parameters controlling the transient behavior. In Figure $6 \mathrm{a}-\mathrm{b}, \Lambda$ is increased from $\Lambda \sim 10^{-10}$ to $\Lambda \sim 10^{-8}$, demonstrating that higher mobility values allow for more horizontal spreading of the biofilm, while the local concentrations of bacteria and EPS are reduced (in this example, there is no nutrient and therefore no net growth of the bacteria or EPS network in the entire biofilm system).

The mixing parameters (i.e. the $\chi_{i j}$ 's) are associated with the overall mixing energies of the various phases. These mixing terms affect the smearing of interfaces and the sorts of structures that develop in the interfacial region between phases. Essentially, when the mixing parameters are small and positive, a larger value of a mixing parameter invites slightly more "smearing" of the interface between two 


\begin{tabular}{|c|c|c|c|}
\hline Symbol & Parameter & Value & Unit \\
\hline $\mathrm{T}$ & Temperature & 303 & Kelvin \\
\hline$\gamma_{1}$ & Distortional energy & $1 \times 10^{7}$ & $\mathrm{~m} \mathrm{~kg} \mathrm{~s}^{-2}$ \\
\hline$\gamma_{2}$ & Strength of the bulk mixing energy & $1 \times 10^{17}$ & $\mathrm{~m} \mathrm{~kg} \mathrm{~s}^{-2}$ \\
\hline$\chi_{s n}$ & Flory-Huggins mixing parameter & $.45-1$ & dimensionless \\
\hline$\chi_{s b}$ & Flory-Huggins mixing parameter & $.45-1$ & dimensionless \\
\hline$\chi_{b n}$ & Flory-Huggins mixing parameter & $.001-.25$ & dimensionless \\
\hline$\lambda$ & Mobility parameter & $1 \times 10^{-8}$ & $\mathrm{~kg}^{-1} \mathrm{~m}^{3} \mathrm{~s}$ \\
\hline$\lambda_{1}$ & polymer relaxation time & $0.1-1000$ & $\mathrm{~S}$ \\
\hline$N_{b}$ & Generalized polymerization index (Bacteria) & $10-100$ & dimensionless \\
\hline$N_{n}$ & Generalized polymerization index (EPS) & $1 \times 10^{3}$ & dimensionless \\
\hline$\mu_{0}$ & Maximum EPS production rate & $1.4 \times 10^{-4}$ & $\mathrm{~kg} \mathrm{~m}^{-3} \mathrm{~s}^{-1}$ \\
\hline$K_{c}$ & Half saturation constant for polymer growth & $1 \times 10^{-4}$ & $\mathrm{~kg} \mathrm{~m} \mathrm{~m}^{-3}$ \\
\hline$C_{1}$ & Maximum EPS decay rate & $.05 \times 10^{-4}$ & $\mathrm{~kg} \mathrm{~m} \mathrm{~m}^{-3}$ \\
\hline$K_{1}$ & Half saturation constant for polymer decay & $1 \times 10^{-4}$ & $\mathrm{~kg} \mathrm{~m}-3$ \\
\hline$C_{2}$ & Maximum bacterial mode growth rate & $4 \times 10^{-4}$ & $\mathrm{~kg} \mathrm{~m} \mathrm{~m}^{-3} \mathrm{~s}^{-1}$ \\
\hline$K_{2}$ & Half saturation constant for bacterial growth & $2 \times 10^{-4}$ & $\mathrm{~kg} \mathrm{~m}-3$ \\
\hline$C_{B}$ & Carrying capacity constant for bacteria & & $\mathrm{kg} \mathrm{m}-3$ \\
\hline$C_{3}$ & Maximum bacterial mode decay rate & $.1 \times 10^{-4}$ & $\mathrm{~kg} \mathrm{~m}^{-3} \mathrm{~s}^{-1}$ \\
\hline$K_{3}$ & Half saturation constant for bacterial decay & $5 \times 10^{-4}$ & $\mathrm{~kg} \mathrm{~m}^{-3}$ \\
\hline$C_{4}$ & Additional bacterial growth rate & 0 & $\mathrm{~kg} \mathrm{~m} \mathrm{~m}^{-3} \mathrm{~s}^{-1}$ \\
\hline$D_{s}$ & Substrate Diffusion constant & $2.3 \times 10^{-9}$ & $\mathrm{~m}^{2} \mathrm{~s}^{-1}$ \\
\hline$D_{d}$ & Disinfectant Diffusion constant & $1.5 \times 10^{-9}$ & $\mathrm{~m}^{2} \mathrm{~s}^{-1}$ \\
\hline$C_{5}$ & Maximum nutrient consumption rate & .1 & $\mathrm{~kg} \mathrm{~m}^{-3} \mathrm{~s}^{-1}$ \\
\hline$K_{5}$ & Half saturation constant for nutrient uptake & $5 \times 10^{-4}$ & $\mathrm{~kg} \mathrm{~m}-3$ \\
\hline$C_{31}$ & Maximum drug consumption rate by bacteria & $2.5 \times 10^{-4}$ & $\mathrm{~kg} \mathrm{~m}-3$ \\
\hline$C_{32}$ & Maximum drug consumption rate by EPS & $1.5 \times 10^{-4}$ & $\mathrm{~kg} \mathrm{~m}^{-3}$ \\
\hline$\eta_{p}$ & Viscosity of the EPS network & 4.3 & $\mathrm{~kg} \mathrm{~m} \mathrm{~m}^{-1} \mathrm{~s}^{-1}$ \\
\hline$\eta_{s}$ & Viscosity of the solvent & $1.002 \times 10^{-3}$ & $\mathrm{~kg} \mathrm{~m} \mathrm{~m}^{-1}$ \\
\hline$\eta_{b}$ & Viscosity of the bacteria in solvent & $4.3 \times 10^{2}$ & $\mathrm{~kg} \mathrm{~m} \mathrm{~m}^{-1} \mathrm{~s}^{-1}$ \\
\hline$\rho_{n}$ & Network density & $1 \times 10^{3}$ & $\mathrm{~kg} \mathrm{~m}-3$ \\
\hline$\rho_{s}$ & Solvent density & $1 \times 10^{3}$ & $\mathrm{~kg} \mathrm{~m}-3$ \\
\hline$\rho_{b}$ & Bacteria density & $1 \times 10^{3}$ & $\mathrm{~kg} \mathrm{~m} \mathrm{~m}^{-3}$ \\
\hline$s$ & Cell volume fraction (per cell) & $.85 \times 10^{-12}$ & $\mathrm{~m}^{3}$ \\
\hline$h$ & Characteristic length scale & $1 \times 10^{-3}$ & $\mathrm{~m}$ \\
\hline$t_{0}$ & Characteristic time scale & 1000 & $\mathrm{~s}$ \\
\hline$f_{0}$ & Characteristic force scale & $1 \times 10^{-15}$ & $\mathrm{~kg} \mathrm{m \textrm {s } ^ { - 1 }}$ \\
\hline
\end{tabular}

TABLE 1. Parameter values used in simulations (unless otherwise specified). Here we have adjusted the growth rates $\mu_{0}, C_{4}, C_{5}$, and $C_{31}$ by dividing by the bacterial volume-fraction constant $s$.

phases than a smaller value since $\chi>0$ promotes mixing while $\chi<0$ promotes demixing in the mixture system. However, this trend reverses when the mixing parameter is positive and relatively large. But, the effect of these mixing parameters is normally small. Note that we generally pick $\chi_{b n}$ to be small (near zero or zero) since the bacteria generally remain fixed within the EPS network (i.e. there are no additional mixing dynamics between the EPS and the Bacterial state other than dissipation). These effects are illustrated in Figure 6c-d. Note in Figure 6c, where a high value of moderate $\chi_{s n}$ results in a more dramatic interface; whereas in Figure $6 \mathrm{~d}$, where a higher value of small $\chi_{s n}$ results in a less dramatic interface, indicating a larger transition/mixing layer between the EPS and the substrate. 
The polymerization indices control the magnitude of the entropic energy for that phase. When the polymerization index is small in a species, the phase of the species tends to spread faster and wider. For instance, in Figure $6 \mathrm{c}$ and d where the polymerization index is set lower for the bacteria $\left(N_{p}=1000, N_{b}=100\right)$, the bacteria can penetrate into the solvent further than the EPS network can conversely. When the value of the polymerization index for the bacteria and the EPS is switched, the bacteria will remain surrounded by the EPS as shown in Figure 7.

Another important parameter in controlling the overall dynamics of the system is the diffusion coefficients for both the nutrient and drug concentrations in the substrate. The timescale associated with the diffusion of the nutrient or drug compared to the timescale on which either is consumed (and thus metabolized) is a key factor determining the dynamics. For example, if diffusion of the nutrients occurs very slowly, while the consumption of the nutrient occurs very rapidly, then the nutrient will not be replaced by diffusion in regions where it is consumed quickly enough to avoid dropping very low. In this scenario, the nutrient would become locally exhausted, stagnating the growth of the biofilm. An alternate scenario, where the drug diffuses quickly, but is consumed relatively slowly, would mean that the drug doesn't get locally exhausted and, thus, given ample supply could sufficiently hinder biofilm growth. Figure 8a-d illustrate the basic features of the dynamics of biofilm growth. When the biofilm is localized (i.e. in Figure $8 \mathrm{a}$ and $8 \mathrm{~b}$ where $\Lambda$ is $\Lambda \sim 10^{-10}$ ), the nutrients are consumed faster than they can be replaced via diffusion. This leads to the spike of growth near the sharp interfacial region between the EPS/Bacteria and the solvent. When the mobility is set higher (Figure $8 \mathrm{c}-\mathrm{d}, \Lambda \sim 10^{-8}$, the biofilm doesn't stay localized, and doesn't locally consume all of the nutrients due to enhanced mixing between the solvent and the rest of the biofilm components. In this scenario, the biofilm grows farther into the solvent, and experiences more evenly distributed growth.

5.2.2. Mixing dynamics in quiescent states. We wish to illustrate basic properties of the dynamics of this three phase mixture. First, consider the case that a bacterial colony has begun to grow in a location, but has not yet formed a biofilm. In this case, nutrients will be fed from the top boundary at $y=1$ at a constant rate of $\left.c\right|_{y=1}=c^{*}$. Figure 9 below shows the initial profile of the bacteria and biofilm volume fraction as well as the profile at $\mathrm{t}=100$. Also, the drug and nutrient concentrations are tracked.

Notice in figure 9 that the bacteria colony has grown in the presence of nutrients, and has also begun to form a biofilm around the bacterial colony signified by the presence of EPS around the bacteria. If time is allowed to progress, and the bacterial colony can grow uninhibited, the biofilm will engulf the entire domain eventually. In this case, the average velocity is zero. However, the velocity for each component is not and their values are depicted in the figure. We note that the velocity for the bacteria is the slowest due to his heavily viscous property. When EPS grows in the biofilm region, the effective solvent is squeezed out of the region which is evidenced by the positive excessive solvent velocity to the right.

We now track the development of a bacterial colony in the presence of a disinfectant or drug which can kill off the bacteria. Here, the disinfectant has two properties. First, it kills the bacteria at a rate proportional to the level of drug in a nonlinear fashion (the Michaelis-Menton kinetics). Secondly, chemicals in the drug cause the polymer network to break down (also in the form of the MichaelisMenton kinetics). Either of these two effects can be tuned to simulate a variety 
of drug therapies, including antibiotics which affect only the bacterial phase, or a disinfectant solution which simultaneously breaks down the biofilm network and sterilizes the surface (kills the bacteria). There are a wide variety of choices for how to administer the drug/disinfectant. Here, the drug is administered in large doses every $t=t_{\text {dose }}$ units of time until $t=100$. The boundary condition for this is set by a piecewise periodic function and the first dose is given at $t=0$. The boundary condition for the drug is a Dirichlet condition, namely,

$$
d(y=1, t)=d_{\text {dose }} \times\left\{\begin{array}{lll}
1, & {\left[\frac{t}{t_{\text {dose }}}\right]} & \text { even }, \\
0, & {\left[\frac{t}{t_{\text {dose }}}\right]} & \text { odd },
\end{array}\right.
$$

where $d_{\text {dose }}$ is the total size of the dose and $t_{\text {dose }}$ is the length of time the dose is given over. Usually, the value of $t_{\text {dose }}$ is small. When drug is administered, the population of the bacterial colony is significantly smaller than it was initially shown in Figure 9 , and the biofilm growth has been slowed greatly. The nutrient concentration in the biofilm shows a slightly nonlinear behavior while outside the biofilm is a simple nearly steady linear profile. The drug concentration is essentially a steady linear profile in this case. It could be altered significantly in other situations as we will show below.

Drug or disinfectant can impose a significant effect to the growth of the EPS and in particular the bacterial population. The effect is inhomogeneous in the biofilm colony normally. Figure 10 depicts the volume fraction of the EPS and the bacteria at time $t=0$ and 100 , respectively, and also the time-dependent values of the two volume fractions at three selected locations, deep inside the biofilm $(\mathrm{y}=0)$, half way in the middle $(y=0.09)$, and at the interface $(y=0.18)$. The competition between the killing off of the bacteria reproduction and EPS growth is contrasted. The effect is most significant at the interface, where the availability of the nutrient makes the growth of the bacteria and EPS production possible during the time period when the drug is not administered leading to a declining seesaw pattern in the time dependent volume fraction profile.

A variety of initial profiles can be modeled, for example biofilm layers could form with a fluid interface between the two. In this case, the bacterial layer closest to the source of the nutrient will grow fastest, but will also be more susceptible to drugs/disinfectants introduced at the upper interface. A simulation of these effects is shown in Figure 11. This gives a gleams of what could happens within a heterogeneous biofilm when nutrient and drugs are diffused.

5.2.3. Sheared Biofilms. In the previous numerical study, we examined the growth and decay of biofilm under the influence of drug/nutrient concentration with the assumption that the average velocity is zero. This assumption is valid in the case where no external shear is applied. For sheared biofilms, we now consider the effect of the velocity coupled to stress and other hydrodynamic variables in several initial boundary value problems.

We consider a 1-D model of a growing biofilm under the influence of a simple shear, i.e., $v_{x}(t, H)=v_{0}$ and $v_{x}(t, 0)=0$. To consider the sheared biofilm, one must first analyze the time scales involved in the shear flow relative to various other time scales. For viscoelastic EPS network, a reasonable relaxation time for the polymer strand may be $\lambda_{1}=0.1 \sim 10$ seconds. Indeed, if we choose such a relaxation time, then the Deborah Number defined by $\Lambda_{1}=\lambda_{1} / t_{0}$, for the case where $t_{0}=1000$ seconds (a reasonable timescale for biofilm growth), is unreasonably small. If we 
couple this with the observation that on this timescale the Reynolds number $R e_{p}$ is very small and $R e_{p} \Lambda_{1} \ll 1$, equation 3.4 can be treated via an asymptotic expansion about this small parameter, and the resulting lowest order dynamics are purely viscous. Physically, this means that picking such a long timescale relative to a short relaxation time, doesn't allow us to see any of the viscoelastic dynamics, and that the dominant dynamics are purely viscous.

In order to observe viscoelastic dynamics, we make our choice of characteristic timescale for a more reasonable Deborah number i.e. $t_{0}=1$ seconds. We note that, on this timescale, the dissipation of the biofilm due to mixing as well as the biofilm growth will be very small. The dominating dynamics are molecular response to the imposed shear and slight internal mixing. In this case, we consider sudden onset of shear after a biofilm has already grown or evolved for a significant period of time. The result of this simulation is given in Figure 12. Note that the velocity of each component of the fluid is tracked (VN Model) and the stress terms are those associated with their corresponding velocity term, and only the elastic contribution to the stress tensor is plotted. The elastic shear stress along with the normal stress components build up in the interior of the biofilm. The shear stress decreases drastically at the biofilm-solvent interface while the normal stress difference undergoes a drastic increases at the interface. The velocity converges to a linear profile outside the biofilm and a nearly linear profile within the biofilm with a sharp transition at the interface.

Next, we consider the case of a purely viscous biofilm, equivalent to picking a much longer experimental timescale than that associated with the EPS relaxation time. In this regime, the polymer network relaxation time is so small relative to $t_{0}$ that we neglect it so that the stress constitutive equation degenerates into $\tau_{e}=$ $\frac{2}{R e_{p}} \mathbf{D}$ (VA model) or $\tau_{e}=\frac{2}{R e_{p}} \mathbf{D}_{n}$ (VN model). Then, the biofilm behaves according to the dynamics of a multi-component viscous fluid. The primary difference between this limit and the viscoelastic fluid is in the stress, namely differences in the shear stress (which is a purely viscous stress in this limit), and the disappearance of the normal stress terms. Figure 13 shows a simulation of a relatively large shear in the viscous regime. In contrast to the elastic regime, the velocity $v_{x}$ is near zero within the biofilm region and linear in the solvent. There is a fairly sharp transition in the interfacial region. The lack of elasticity leads to non-compliance of the biofilm region from the solvent region. The shear stress in the polymer network exhibits a large fluctuation at the biofilm-solvent interface, which is absent in the viscoelastic regime in small time scales. This behavior in the viscous regime can be justified by the difference of the time scale on which the behavior is observed.

5.2.4. Impermeable Networks. A more realistic model should perhaps accounts for the fact that an EPS network affects the rate of nutrient and drug diffusion in the biofilm. Indeed, one reason that biofilms are difficult to treat is that it is difficult to get drugs/disinfectants through the EPS network to where it can destroy the bacterial colonies. One possible way to incorporate this is to have the diffusion coefficients be functions of the amount of solvent present:

$$
D_{s}=D_{s}^{0} \phi_{s}^{\alpha}, D_{d}=D_{d}^{0} \phi_{s}^{\beta} .
$$

This is a more generalized diffusion equation, where $\alpha, \beta=0$ is the default model, while $\alpha, \beta \gg 1$ would mean a highly inpermeable biofilm layer. To illustrate the profound effect this sort of models can have on the overall dynamics of the biofilm system, the figure below shows what happens in the default case and then a case 
where the biofilm layer is very inpermeable to the drug, but still allows nutrients into the colony. As the power $\beta$ increases, the volume fraction for the bacteria reduces sizably shown in Figure 14. As the diffusivity for the drug reduces, the drug concentration outside the biofilm increases while the one deep inside the biofilm drops drastically.

The model discussed above is a qualitative attempt of adding the impermeability of EPS networks to the model, and the viability of this model of diffusivity (as well as the powers and coefficients involved) must be determined through experimental observation. Hinson et. al. [13] have considered a model of effective biofilm diffusivities inspired by biofilm kinetics which has been fit to experimental data. The effective diffusivity within the biofilm can be related to our phase field model according to the equation:

$$
D_{e f f}=\frac{2 D_{d}\left(1-\phi_{b}\right) \phi_{s}}{\left(2+\phi_{b}\right)\left(\phi_{s}+\phi_{n} / D_{p r}\right)} .
$$

Here, the parameter $D_{p r}$ is an empirical parameter that must be fit by experimental observation (it is generally much smaller than the diffusion parameter within the solvent). Some quick analysis of the above equation shows that the effective diffusivity goes to the default value of $D_{d}$ when the biofilm concentration is small $\left(\phi_{n}, \phi_{b} \ll 1\right)$, and goes to zero quickly as the biofilm volume fraction increases. Assuming that $\phi_{n}=2 \phi_{b}$, and for $D_{p r}=.02$, we give a table of sample effective diffusivities, and seamlessly integrate this diffusivity model into our multicomponent biofilm model.

\begin{tabular}{l|l} 
Biofilm Volume Fraction (\%) & Effective Diffusivity \\
\hline 0 & $D_{d}$ \\
.01 & $.744 D_{d}$ \\
.025 & $.533 D_{d}$ \\
.05 & $.354 D_{d}$ \\
.1 & $.202 D_{d}$ \\
.2 & $.097 D_{d}$ \\
\hline
\end{tabular}

Note that as the biofilm volume fraction $\left(\phi_{n}+\phi_{b}\right)$ increases from 0 percent to 20 percent, the diffusivity within the fluid drops from its default value, to less than 10 percent of the default value. These values are consistent with those found in other studies [13]. A figure of a growing biofilm with this type of effective diffusivity is given in Figure 15.

6. Conclusion. We have developed a hydrodynamical theory for biofilm-bulk fluid mixture by modeling the EPS network, bacteria and the effective solvent (consisting of solvent and all nutrient materials) explicitly. This theory is formulated using the single fluid multicomponent paradigm so that an incompressible average velocity field is prominently identified while the individual velocity for each individual component is given by a combination of the mixing kinetics and the average velocity. Two linear growth modes are identified associated with the growth mechanisms caused by the EPS production and the bacterial growth. Their presence is determined by the model parameters. Numerical investigations of the model for transient dynamics in one dimensional space are carried out. The various concentration dependent diffusivities for the nutrient and the drug are numerically tested demonstrating the profound impact that they have on the internal growth of biofilms 
and distribution of the nutrient and drugs.

\section{REFERENCES}

[1] G. C. Barker and M. J. Grimson, A cellular automaton model of microbial growth, Binary Comput. Microbiol., 5 (1993), 132-137.

[2] E. Ben-Jacob, O. Schochet, A. Tenenbaum, I. Cohen, A. Czirok and V. Tamas, Generic modelling of cooperative growth patterns in bacterial colonies, Nature(London), 368 (1994), $46-49$.

[3] A. N. Beris and B Edwards, "Thermodynamics of Flowing System," Oxford University Press, 1994.

[4] J. W. Cahn and J. E. Hilliard, Free energy of a nonuniform system. I: Interfacial free energy, J. Chem. Phys., 28 (1958), 258-267.

[5] J. W. Cahn and J. E. Hilliard, Free energy of a nonuniform system-iii: Nucleation in a 2-component incompressible fluid, J. Chem. Phys., 31 (1959), 688-699.

[6] N. G. Cogan and J. P. Keener, The role of the biofilm matrix in structural development, Math. Med. Biol., 21 (2004), 147-166.

[7] R. L. Colasanti, Cellular automata models of microbial colonies, Binary Comput. Microbiol., 4 (1992), 191.

[8] J. D. Dockery and I. Klapper, Finger formation in biofilm layers, SIAM. J. Appl. Math., 62 (2001), 853-869.

[9] H. J. Eberl, D. F. Parker and M. C. M. van Loosdrecht, A new deterministic spatio-temporal continuum model for biofilm development, J. Theor. Mde., 3 (2001), 161-175.

[10] P. J. Flory, "Principles of Polymer Chemistry," Cornell University Press, Ithaca, NY, 1953.

[11] D. R. Noguera G. Pizarro and D. Griffeath, Quantative cellular automaton model for biofilms, J. Environ. Eng., 127 (2001), 782-789.

[12] S. W. Hermanowicz, A simple 2d biofilm model yields a variety of morphological features, Math. Biosci., 169 (2001), 1-14.

[13] R. K. Hinson and W. M. Kocher, Model for effective diffusivities in aerobic biofilms, Journal of Environmental Engineering, (1996), 1023-1030.

[14] J. C. Kissel, P. L. McCarty and R. L. Street, Numerical simulation of mixed-culture biofilm, J. Environ. Eng., 110 (1984), 393-411.

[15] I. Klapper and J. Dockery, Role of cohesion in material description of biofilms, Phys. Rev. E, 74 (2006), 031902 .

[16] C. Picioreanu, M. C. Loosdrecht and J. J. Heijnen, Discrete-differential modelling of biofilm structure, Wat. Sci. Tech., 39 (1999), 115-122.

[17] C. Picioreanu, M. C. Loosdrecht and J. J. Heijnen, Effect of diffusive and convective substrate transport on biofilm structure formation: a two-dimensional modeling study, Biotech. Bioeng., 69 (2000), 504-515.

[18] C. Picioreanu, M. C. Loosdrecht and J. J. Heijnen, Two-dimensional model of biofilm detachment caused by internal stress from liquid flow, Biotech. Bioeng., 72 (2001), 205-218.

[19] C. Picioreanu, M. C. M. Loosdrecht and J. J. Heijnen, A new combined differential-discrete cellular automaton approach for biofilm modeling: Application for growth in gel beads, Biotech. Bioeng., 57 (1998), 718-731.

[20] G. Pizarro, R. Moreno C. Garcia and M. E. Sepulveda, Two-dimensional cellular automaton model for mixed-culture biofilm, Wat. Sci. Tech., 49 (2004), 193-198.

[21] B. E. Rittmann, The effect of shear stress on biofilm loss rate, Biotech. Bioeng., 24 (1982), 501-506.

[22] B. E. Rittmann and P. L. McCarty, Evaluation of steady-state biofilm kinetics, Biotech. Bioeng., 22 (1980), 2359-2373.

[23] B. E. Rittmann and P. L. McCarty, Model of steady-state-biofilm kinetics, Biotech. Bioeng., 22 (1980), 2243-2357.

[24] Q. Wang and T. Zhang, Review of mathematical models for biofilms, Communication in Solid State Physics, 2010.

[25] O. Wanner and W. Gujer, Competition in biofilms, Wat. Sci. Tech., 17 (1984), 27-44.

[26] O. Wanner and W. Gujer, A multispecies biofilm model, Wat. Sci. Tech., 28 (1986), 314-328.

[27] J. W. T. Wimpenny and R. Colasanti, A unifying hypothesis for the structure of microbial biofilms based on cellular automaton models, FEMS Micro. Ecol., 22 (1997), 1-16. 
[28] T. Zhang, N. Cogan and Q. Wang, Phase-field models for biofilms I. theory and simulations, SIAM, J. Appl. Math., 69 (2008), 641-669.

[29] T. Zhang, N. Cogan and Q. Wang, Phase-field models for biofilms II. 2-d numerical simulations of biofilm-flow interaction, Commun. Comput. Phys., 4 (2008), 72-101.

Received December 2009; revised March 2010.

E-mail address: blindley@nlsleah.nrl.navy.mil

E-mail address: qwang@math.sc.edu

E-mail address: zhang@math.montana.edu 

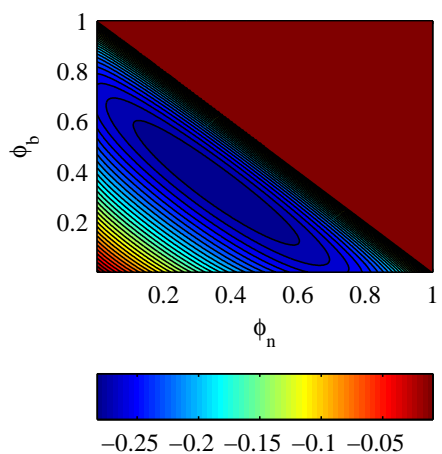

(a) $N_{p}=20, N_{b}=20, \chi_{b n}=0$
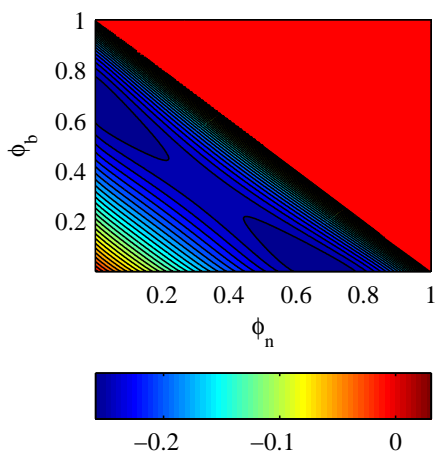

(c) $N_{p}=20, N_{b}=20, \chi_{b n}=.3$

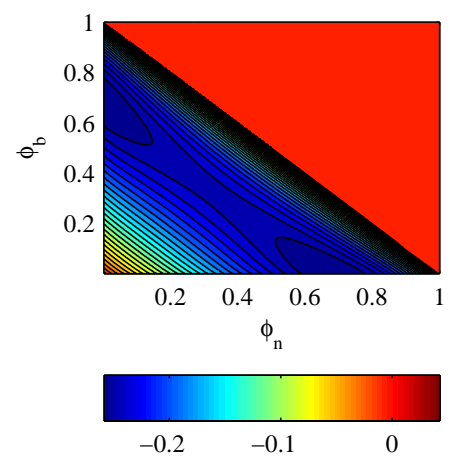

(e) $\chi_{b n}=.35, \chi_{s b}=.55, \chi_{s n}=.55$

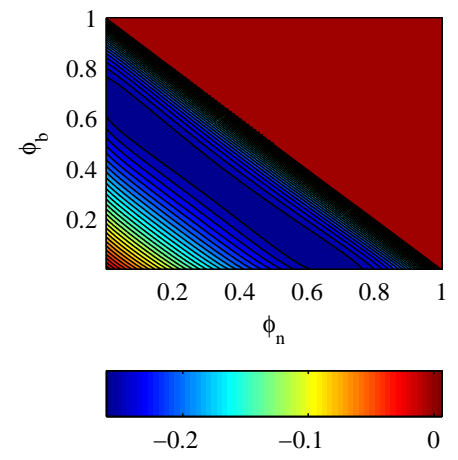

(b) $N_{p}=20, N_{b}=20, \chi_{b n}=.2$

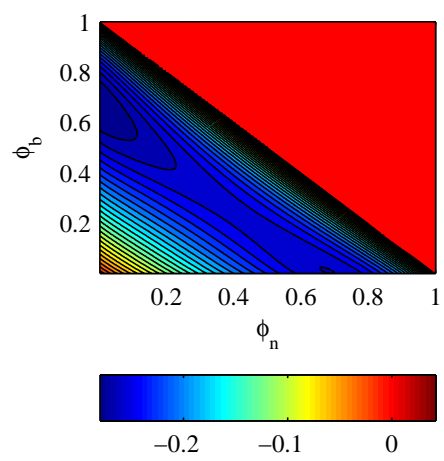

(d) $\chi_{b n}=.35, \chi_{s b}=.55, \chi_{s n}=.45$

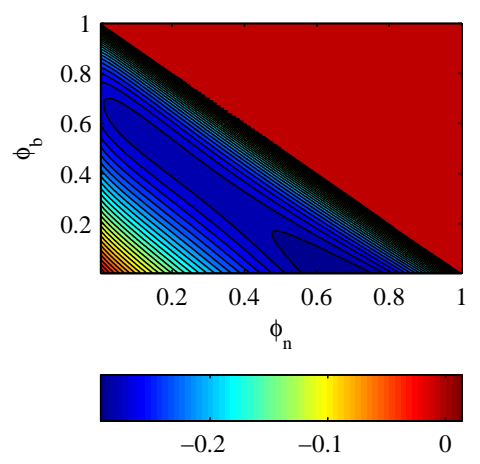

(f) $\chi_{b n}=.35, \chi_{s b}=.45, \chi_{s n}=.55$

FiguRE 1. The normalized bulk mixing energy density at two sequences of selected mixing parameters. Notice that as $\chi_{b n}$ is increased in Figures (a)-(c), the single well gradually stretches then pinches off into two separate wells. In Figures (d)-(f), changing the balance between $\chi_{s b}$ and $\chi_{s n}$ increases the depth of the double well potential near one of the wells. If $\chi_{s b}>\chi_{s n}$, the well near the $\phi_{b}$ axis deepens, and vice-versa. Here $N_{b}=N_{p}=20$, and $\Gamma_{11}=\Gamma_{12}=\Gamma_{13}=41.8337, \Gamma_{2}=418337$ and the energy is normalized by the constant $\Gamma_{11} \times \Gamma_{2}$. 


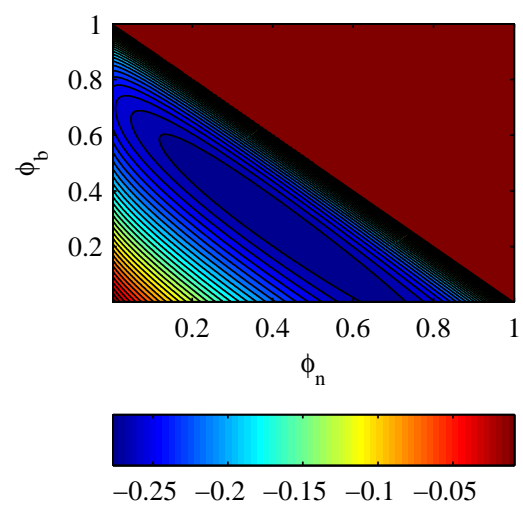

(a) $N_{b}=10, N_{p}=1000$.

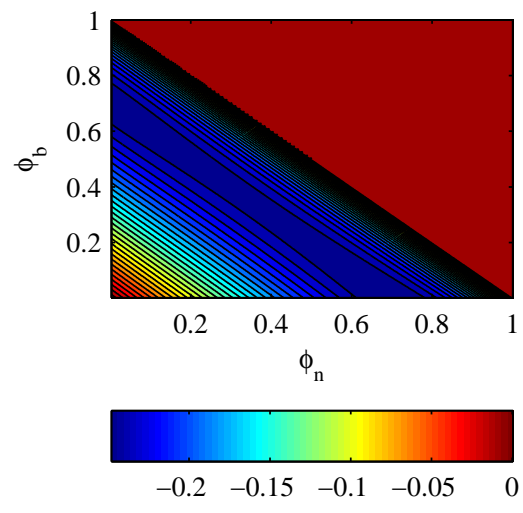

(b) $N_{b}=100, N_{p}=1000$.

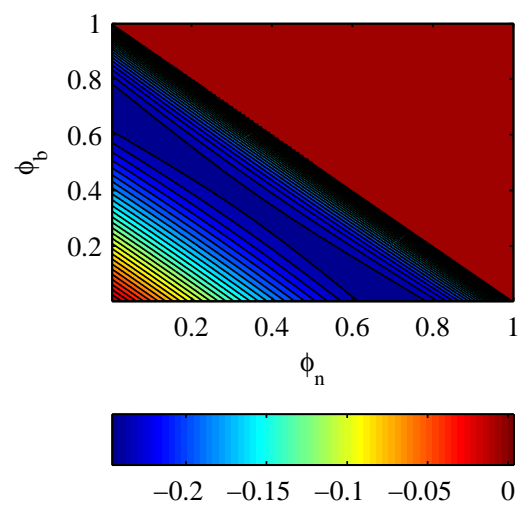

(c) $N_{b}=1000, N_{p}=1000$.

FIGURE 2. The normalized bulk mixing energy density $\hat{f}\left(\phi_{n}, \phi_{b}\right)$ at several effective polymerization indices for the bacteria. Here the mixing parameters are the same in all three calculations, $\chi_{b n}=.05$, $\chi_{s n}=.55, \chi_{s b}=.55$, and these values will be used in simulations later. The effect of adjusting the effective polymerization index for the bacterium, relative to the EPS, is to stretch the single well into two wells. 


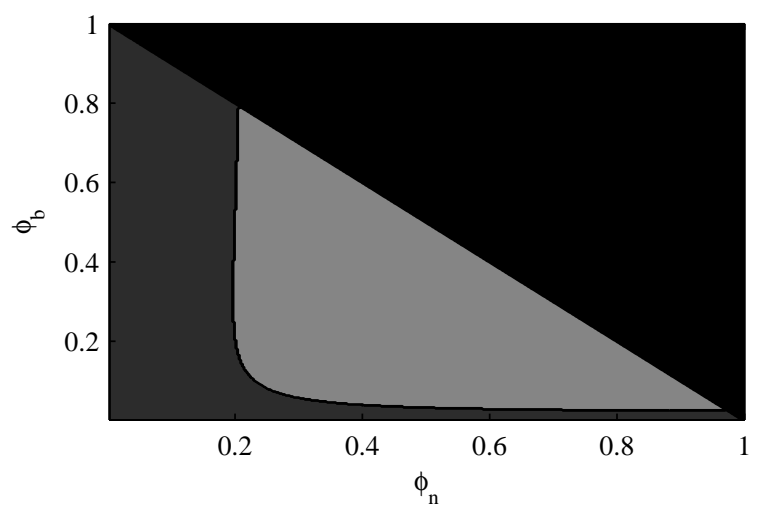

(a) $\chi_{b n}=.25 \chi_{s n}=.45 \chi_{s b}=.45 N_{b}=10 N_{p}=100$

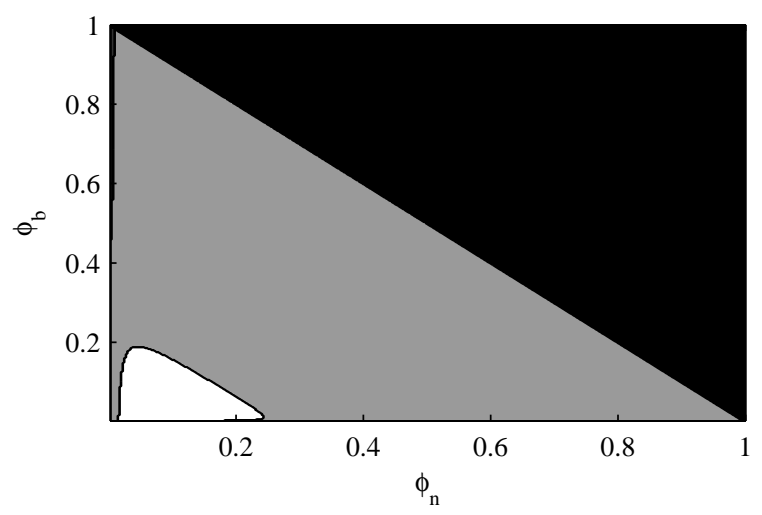

(b) $\chi_{b n}=.65 \chi_{s n}=.85 \chi_{s b}=.85 N_{b}=100 N_{p}=1000$

FIgURE 3. Portrait of two representative stability scenarios in phase space. Here, the black region is outside of the domain, the dark grey in (a) corresponds to both eigenvalues negative, the light grey in both figures corresponds to one positive eigenvalue, and the white in (b) corresponds to both eigenvalues positive. 

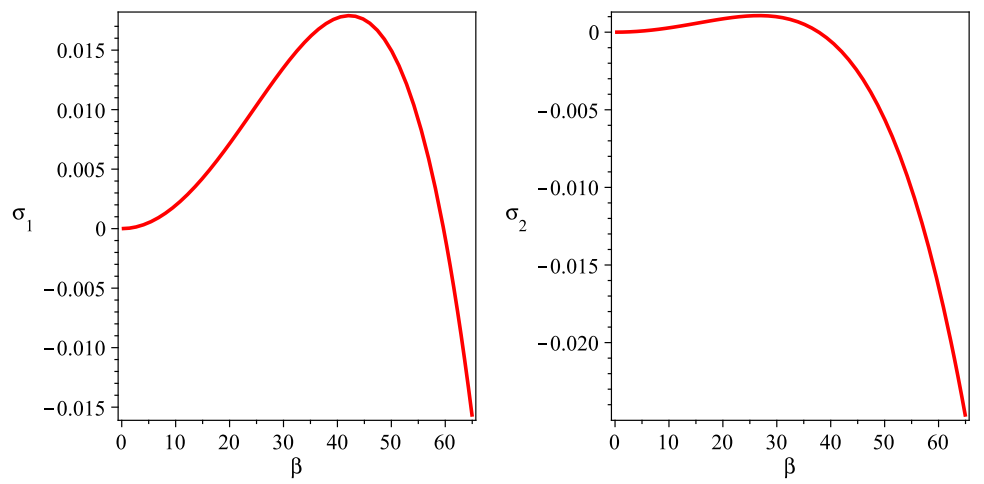

(a) Long wave instability in both growth rates when $\chi_{b n}=.65, \chi_{s n}=.85$ $\chi_{s b}=.85, N_{b}=1000, N_{p}=10000$ and $\phi_{n}=.05, \phi_{b}=.05$
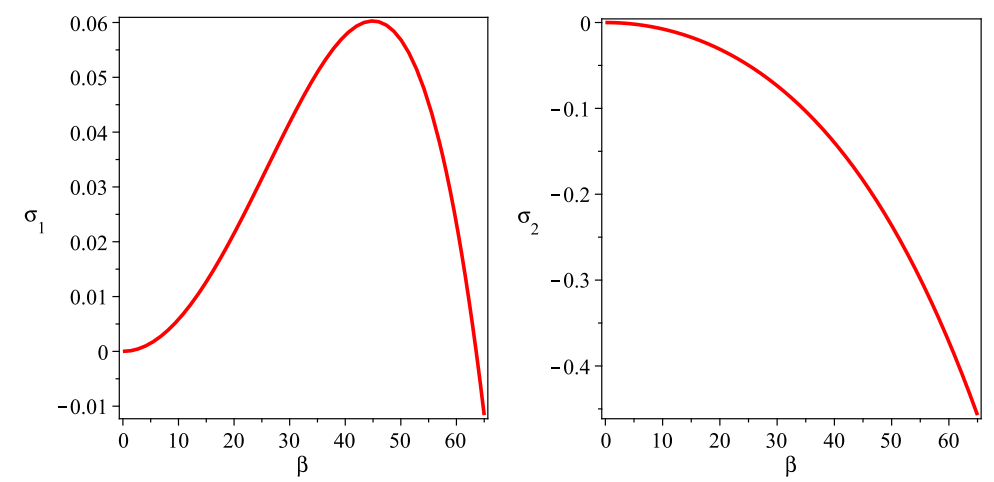

(b) Long wave instability in one growth rate when $\chi_{b n}=.65, \chi_{s n}=.65$ $\chi_{s b}=.65, N_{b}=100, N_{p}=1000$ and $\phi_{n}=.19, \phi_{b}=.19$
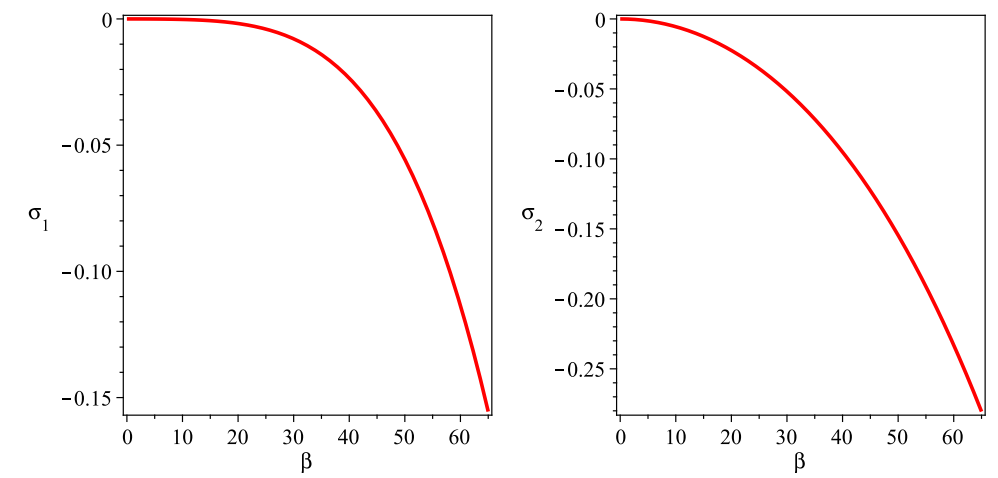

(c) Long wave stability in the both growth rates when $\chi_{b n}=.45, \chi_{s n}=.45$ $\chi_{s b}=.45, N_{b}=10, N_{p}=100$ and $\phi_{n}=.1, \phi_{b}=.05$

FiguRE 4. The linearized growth rates for the governing system of equations in three representative cases as a function of wave number $\beta$ for $c_{0}=d_{0}=0$. In all cases, $\Gamma_{11}=\Gamma_{12}=\Gamma_{13}=41.8337$, $\Gamma_{2}=418337$. 


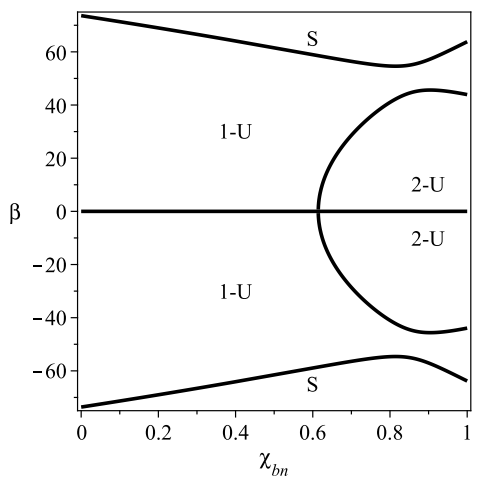

(a) $\chi_{s b}=.85, \chi_{s n}=.85$

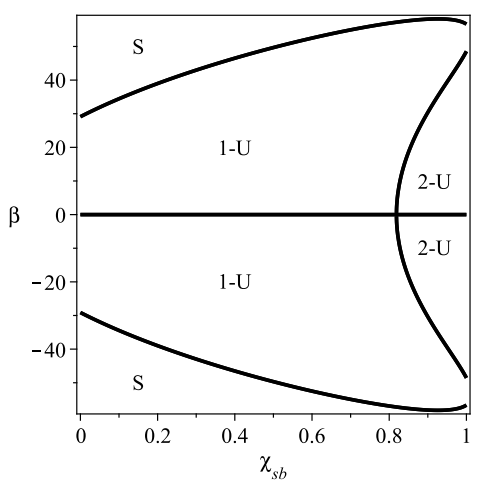

(c) $\chi_{b n}=.65, \chi_{s n}=.85$

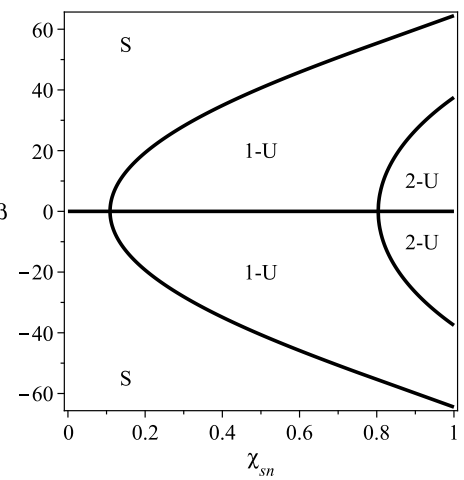

(b) $\chi_{b n}=.65, \chi_{s b}=.85$

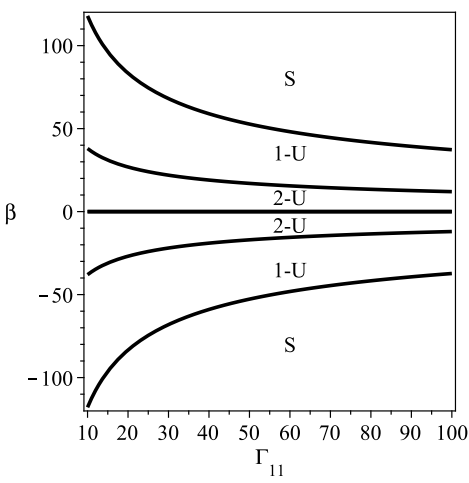

(d) $\Gamma_{2}=418337$

Figure 5. The cutoff wave number that separates the stable from the unstable eigenmodes as a function of the mixing parameters and $\Gamma_{11}$, respectively. The regions are labeled as S: two stable eigenmodes, 1-U: one unstable eigenmode, and 2-U: 2 unstable eigenmodes. Here, $N_{b}=100, N_{p}=1000$, and $\Gamma_{11}=\Gamma_{12}=\Gamma_{13}=$ 41.8337, $\Gamma_{2}=418337$ with $\phi_{n}^{0}=.05$ and $\phi_{b}^{0}=.05$. (a-c) The parameter values are listed in the subtitle. (d). The parameter values are $\chi_{b n}=.65, \chi_{s n}=.85$ and $\chi_{s b}=.85, N_{b}=100, N_{p}=1000$, and $\Gamma_{2}=418337$. 


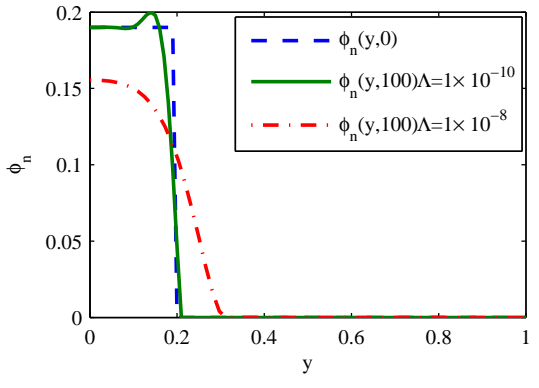

(a) $\Lambda=1 \times 10^{-8}$ or $1 \times 10^{-10}$

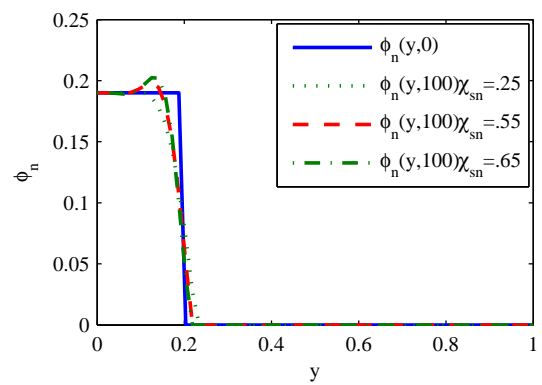

(c) $\chi_{s n}=.25, .55$, or .65

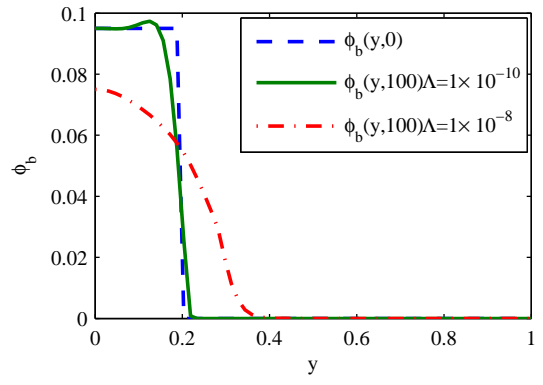

(b) $\Lambda=1 \times 10^{-8}$ or $1 \times 10^{-10}$

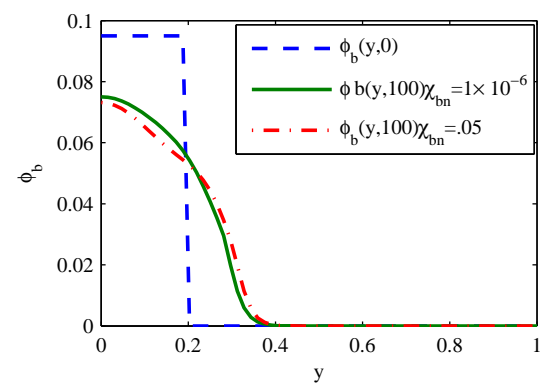

(d) $\chi_{b n}=0$, or .05

Figure 6 . The evolution of the phase variables and the nutrient concentration under various parameter choices. The parameters are given in Table 1 and $N_{p}=1000 N_{b}=100 \chi_{s n}=.55 \chi_{s b}=.55$ $\chi_{n b}=.001 \Lambda=1 \times 10^{-8}, 1 \times 10^{-10}$ unless otherwise specified. (a) and (b) show the effect of increasing $\Lambda$ on the mixing dynamics (no network growth). (c) and (d) show the mild change in the interface associated with increasing the mixing parameter (without the network growth).

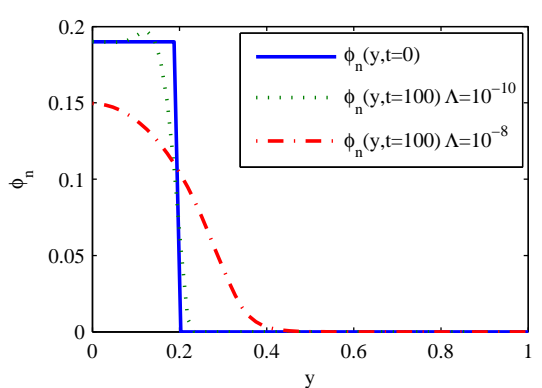

(a)

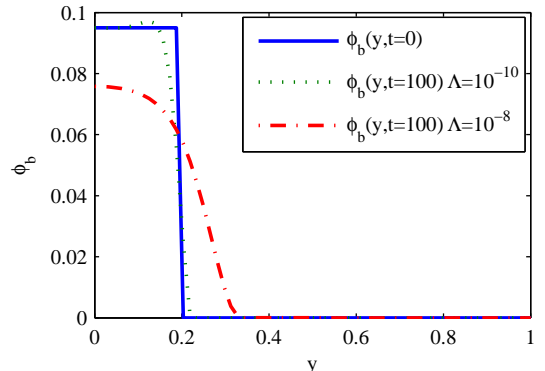

(b)

FiguRE 7. The evolution of the phase variables without the nutrient when $N_{p}<N_{b}$. The bacteria have less entropic energy than the EPS does. So, they remain within the EPS network in contrast to fig. $6(a, b)$. 


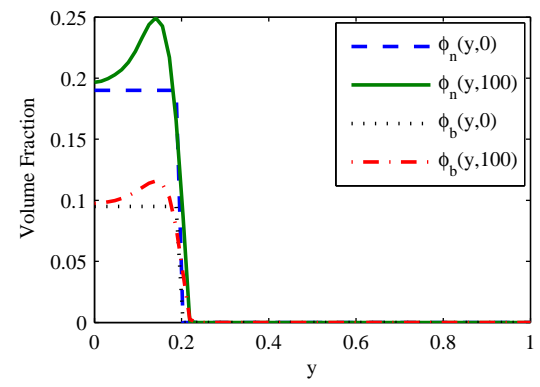

(a) $\Lambda=1 \times 10^{-10}$

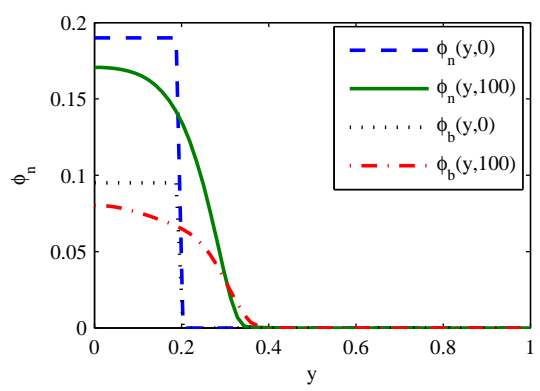

(c) $\Lambda=1 \times 10^{-8}$

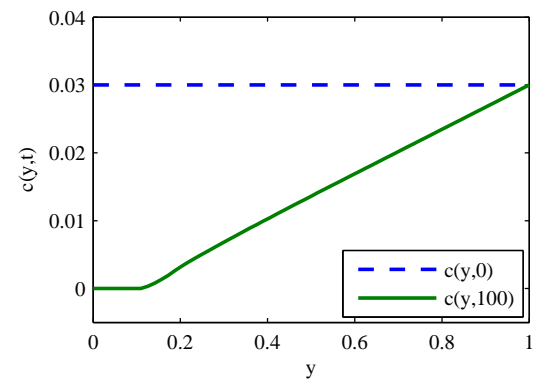

(b) $\Lambda=1 \times 10^{-10}$

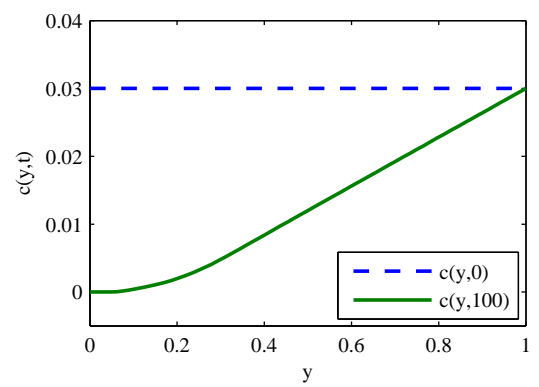

(d) $\Lambda=1 \times 10^{-8}$

Figure 8 . The evolution of the phase variables and the nutrient concentration under various parameter choices. The parameters are given in Table 1 and $N_{p}=1000 N_{b}=100 \chi_{s n}=.55 \chi_{s b}=.55$ $\chi_{n b}=.001 \Lambda=1 \times 10^{-8}, 1 \times 10^{-10}$ unless otherwise specified. These figures show biofilm and nutrient profiles associated with bacterial and network growth under different choices of $\Lambda$. 

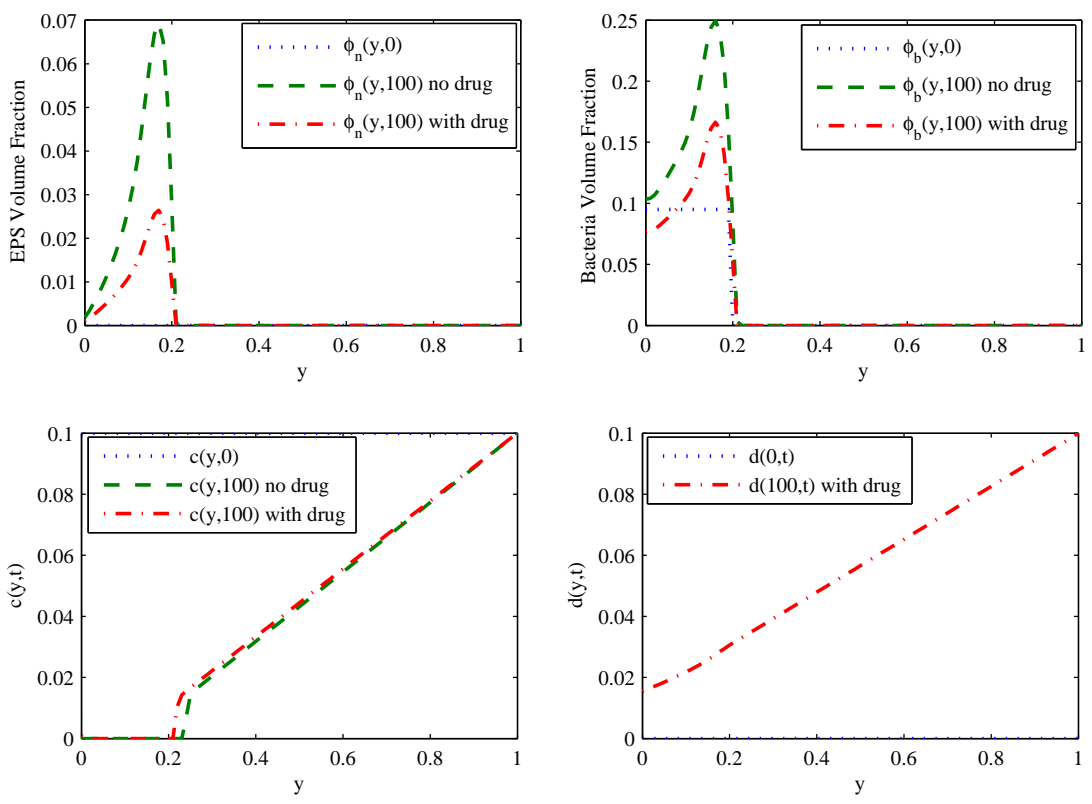

FiguRE 9. The development of a biofilm in a region of a bacterial colony both with and without a drug/disinfectant. The parameter values used here are $N_{p}=1000 N_{b}=100 \chi_{s n}=.55 \chi_{s b}=.55$ $\chi_{n b}=0, \Lambda=1 \times 10^{-10}$, and the other growth parameters are specified in table 1. 


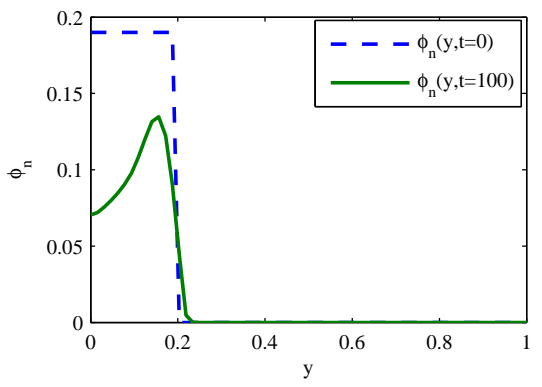

(a) EPS Volume Fraction

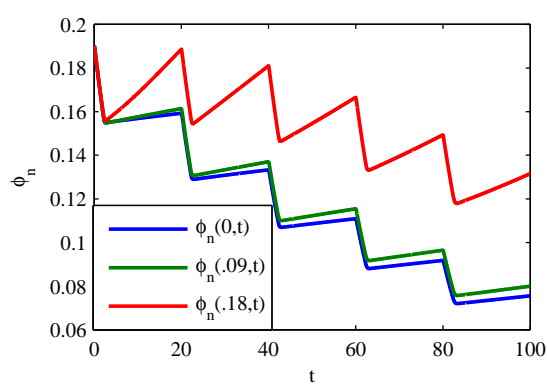

(c) EPS Time Series

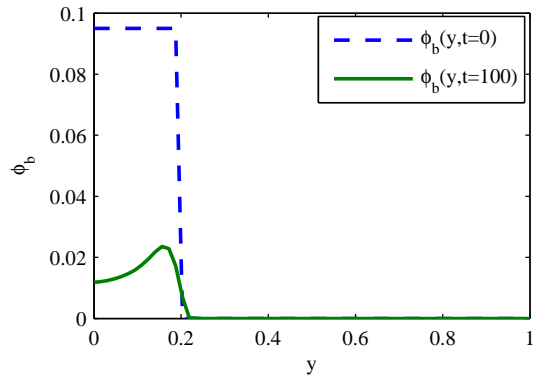

(b) Bacteria Volume Fraction

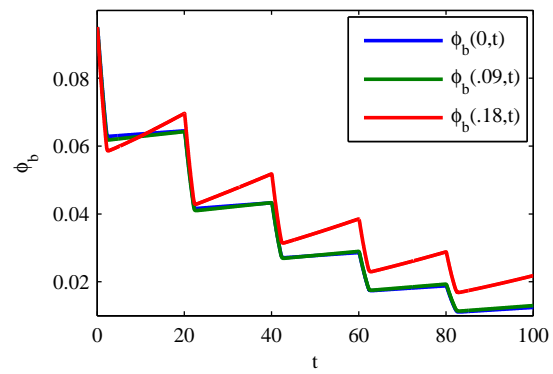

(d) Bacteria Time Series

Figure 10. A growing biofilm under the influence of a drug treatment regimen. Here the drug slowly breaks down the biofilm while quickly killing the bacteria. A large dose $d=10$ is administered at $y=1$ every $t_{\text {dose }}=20$ time units. Here, the nutrient concentration $c(1, t)=.1$ is imposed at the boundary and the parameter choices for the growth rates are those described in table 1 with $\Lambda=1 \times 10^{-10}, \chi_{s n}=\chi_{s b}=.45$, and $\chi_{b n}=0$. The bounce-back growth is more significant after each dosage in the interfacial region than in the biofilm region. The bacterial population decays in general. 


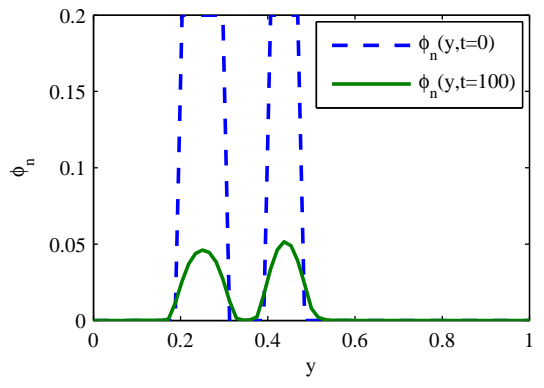

(a) EPS Volume Fraction

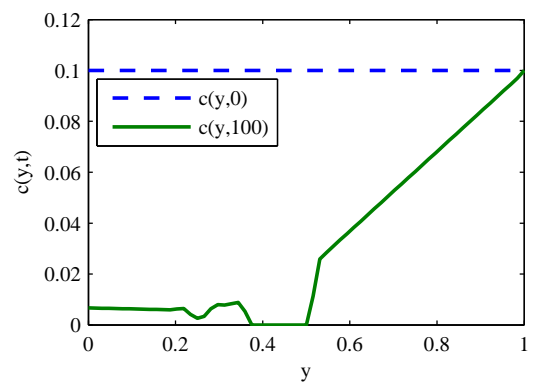

(c) Nutrient Concentration

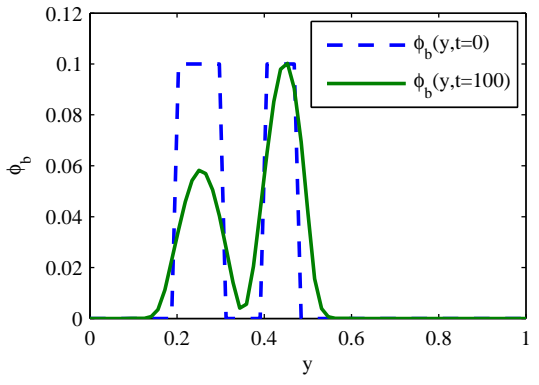

(b) Bacteria Volume Fraction

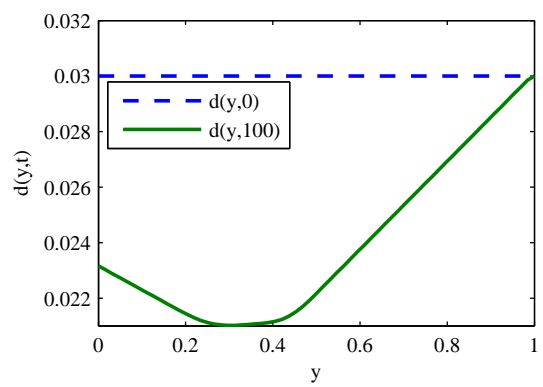

(d) Drug Concentration

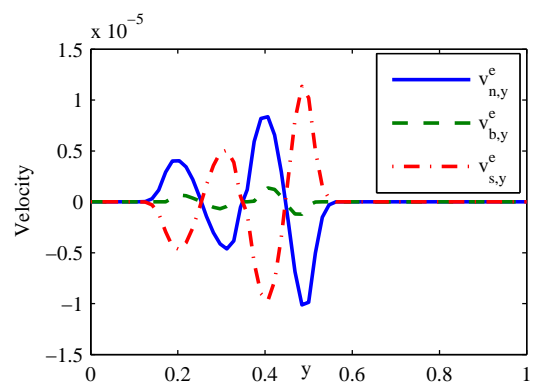

(e) Excessive Velocity

FigURE 11. Evolution of biofilm layers with both nutrient and drug fed in at $y=1$. The nutrient cannot reach the bacterial colony that is further away, inhibiting its growth. The drug concentration is near depleted between the two biofilm colonies. The parameter values used here are $N_{p}=1000 N_{b}=100 \chi_{s n}=.55, \chi_{s b}=.55$, $\chi_{n b}=0, \Lambda=1 \times 10^{-10}$, with the other growth parameters specified in table 1. 


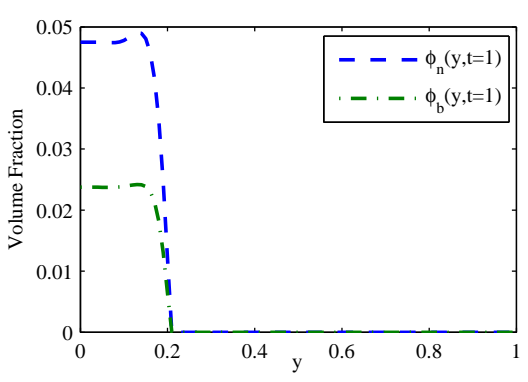

(a) Biofilm Volume Fraction

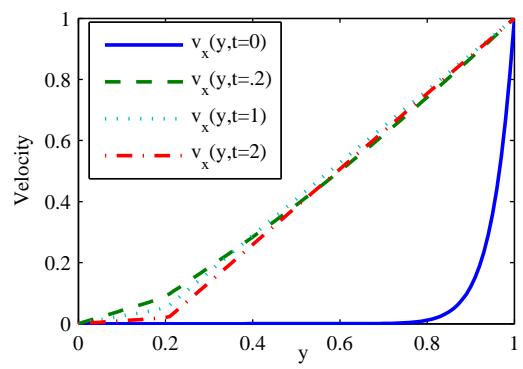

(c) Time Snapshots of Velocity

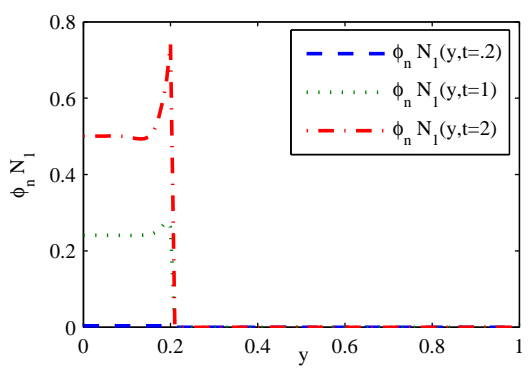

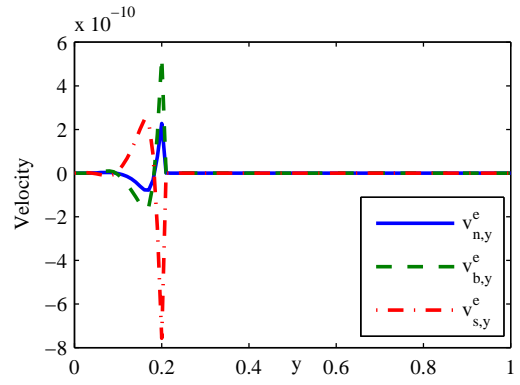

(b) Excessive Velocity

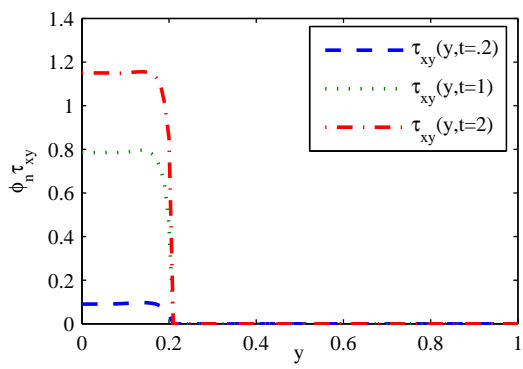

(d) Time Snapshots of Elastic Shear Stress

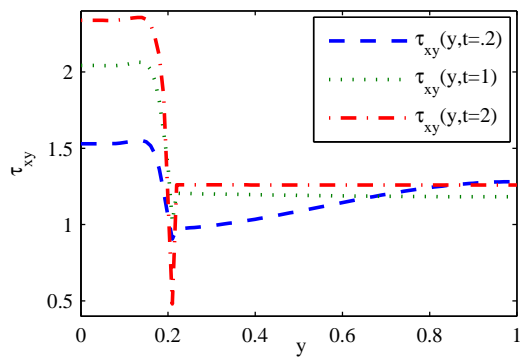

(e) Time Snapshots of Elastic First Normal (f) Time Snapshots of Total Shear Stress Stress Difference

Figure 12. A biofilm under planar shear for $\Lambda=1 \times 10^{-10}$. Here the shear is applied to an already formed biofilm, and the characteristic timescale is picked such that the Deborah number is $D e=1$. The fluid parameters in this timescale are, $R e_{p}=.0023, R e_{b}=.023$ and $R e_{s}=.998$. Several time snapshots are shown which illustrate the pullback of the viscoelastic EPS network against the imposed shear. Because the viscoelastic shear timescale is much shorter than the biofilm growth rate and dissipation timescale, there is negligible evolution of the bacteria/EPS phase variables. The volume fractions used here are smaller than in other simulations to dramatize the viscoelastic response of the fluid. 


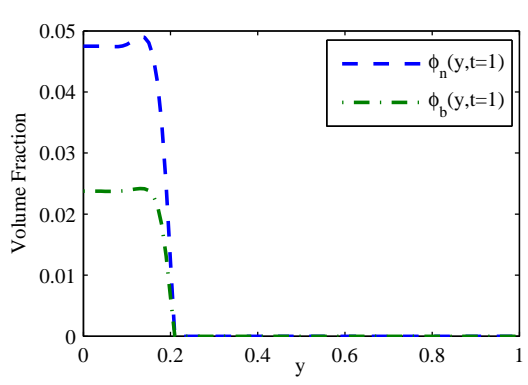

(a) Biofilm Volume Fraction

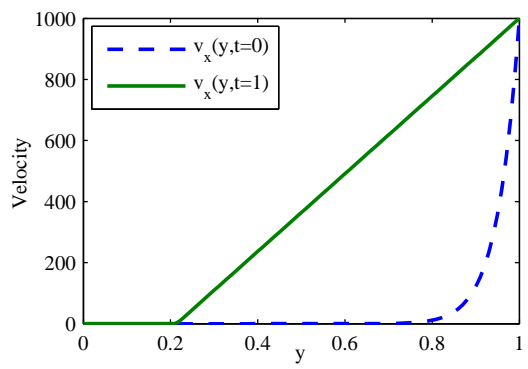

(c) Velocity

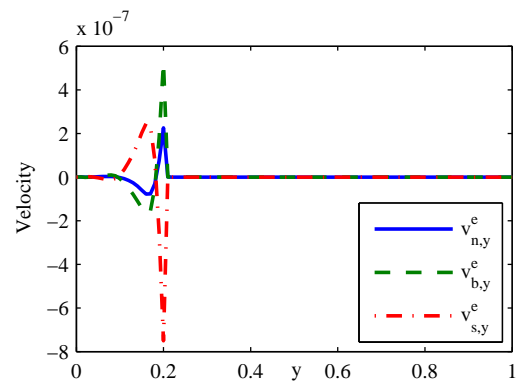

(b) Excessive Velocity

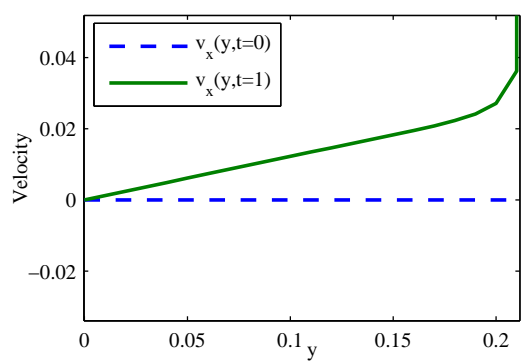

(d) Velocity Inside Biofilm Region

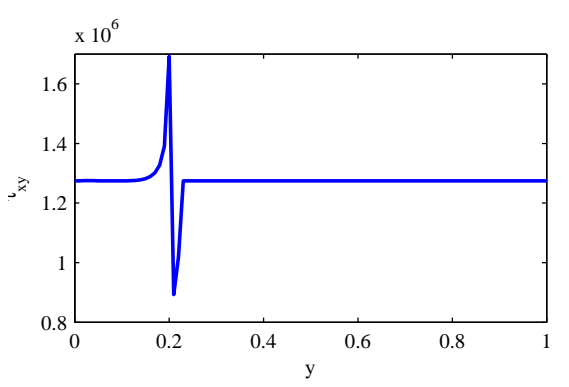

(e) Shear Stress

FiguRe 13. A biofilm under planar shear in the viscous (or long characteristic timescale) regime. Here, the viscous parameters are $R e_{p}=2.33 \times 10^{-7}, R e_{p}=2.33 \times 10^{-9}$ and $R e_{s}=9.98 \times 10^{-4}$ for $\Lambda=1 \times 10^{-10}$. Since this is a relatively large shear and the relaxation time is tiny compared to the characteristic timescale, the simulation is only run until $t=1$ treating the EPS polymer as a viscous fluid. 

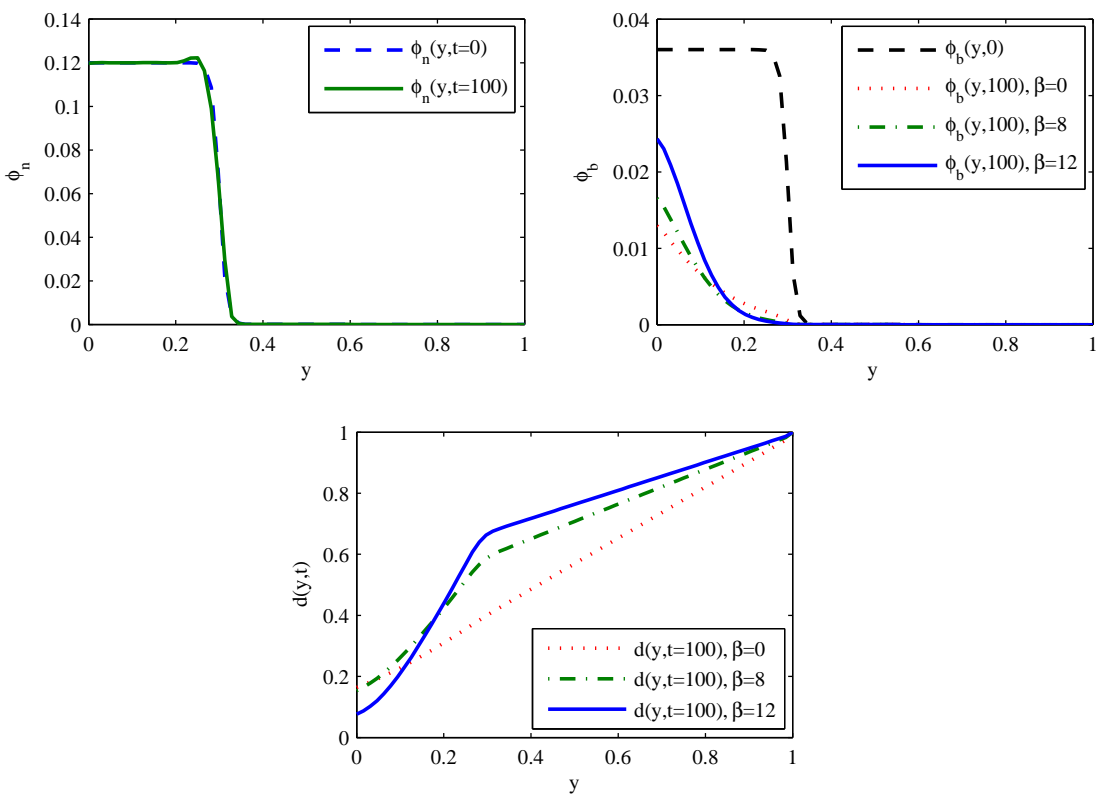

FiguRE 14. Various degrees of permeability of the EPS network. In these simulations, $c=0$ is set to isolate the effects of impermeability, and the drug is assumed to kill only the bacteria without affecting the EPS network. Higher degrees of impermeability insulate the bacterial colony from the drug. The values used here are the standard values used: $N_{p}=1000 N_{b}=100 \chi_{s n}=.55 \chi_{s b}=.55$ $\chi_{n b}=0, \Lambda=1 \times 10^{-10}$.
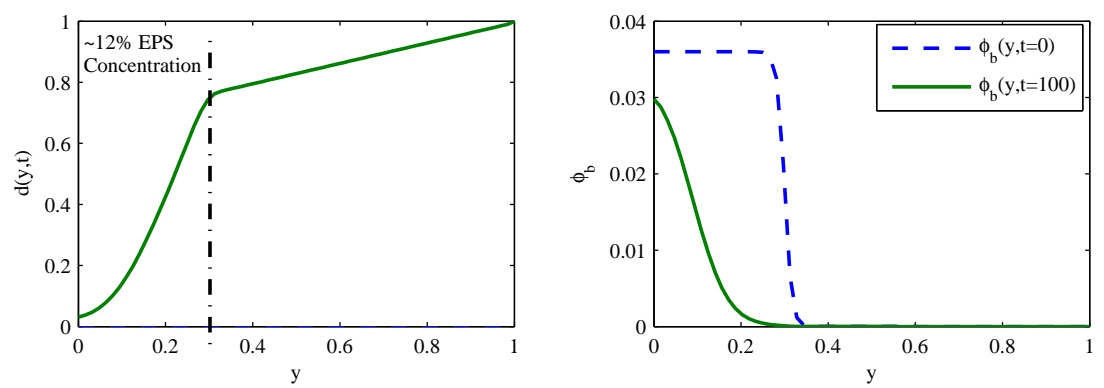

Figure 15. The drug concentration profile at $t=100$ for the Hinson model. The parameters used here are identical to those used in Figure 14. Notice that this model predicts even more impermeability of the EPS network than the power law models and that the bacterial colony is not degraded as much. 